\title{
Hard thermal loop effective action of topologically massive gluons in $3+1$ dimensions
}

\author{
Debmalya Mukhopadhyay, ${ }^{1, *}$ R. Kumar ${ }^{2, \dagger}$ Jan-e Alam, ${ }^{1, \ddagger}$ and Sushant K. Singh ${ }^{1, \S}$ \\ ${ }^{1}$ Variable Energy Cyclotron Centre, 1/AF, Bidhan Nagar, Kolkata-700064, India \\ ${ }^{2}$ Department of Physics and Astrophysics, University of Delhi, New Delhi-110007, India
}

(Received 1 December 2019; accepted 6 April 2020; published 27 April 2020)

\begin{abstract}
An effective action for "soft" gluons has been constructed by integrating out hard thermal modes of topologically massive vector bosons at one loop order. The gluons are equally massive in the non-Abelian topologically massive model due to a quadratic coupling $B \wedge F$ where a 2-form field $B$ is coupled quadratically with the field strength $F$ of the Yang-Mills (YM) field. This non-Abelian model provides the same asymptotic freedom of strong coupling which is found in massless YM theory at zero temperature. The presence of a gauge-invariant infrared cutoff plays the role of magnetic mass. Thus, in the model, it can be used to get the transport coefficients in the perturbative regime.
\end{abstract}

DOI: 10.1103/PhysRevD.101.074039

\section{INTRODUCTION}

Gauge theory plays a crucial role in the standard model of particle physics for the description of fundamental interactions in nature [1-3]. The standard model is the theory that describes three fundamental interactions (i.e., electromagnetic, weak, and strong) among all the known particles, excluding gravitational interaction. In the electroweak sector, global $S U(2) \times U(1)$ symmetry is spontaneously broken to global $U_{\mathrm{em}}(1)$ symmetry. This residual symmetry is responsible for the electromagnetic interaction. The mediators of the weak force, $W^{ \pm}$and $Z$ bosons, become massive via the Higgs mechanism through the process of spontaneous symmetry breaking. The Higgs particle has been discovered in the Large Hadron Collider (LHC) [4,5] recently.

The strong sector in the standard model has a special characteristic that makes it significantly different from the electroweak sector. The elementary particles, quarks and gluons, that interact strongly are not found free in any experiment to date. The dynamics of quarks and gluons is governed by quantum chromodynamics (QCD). The confinement of the quarks within the hadrons is yet to be understood. Besides this, one of the other important features of the strong interaction is the asymptotic freedom, which

\footnotetext{
*debphys.qft@gmail.com

raviphynuc@gmail.com

*jane@vecc.gov.in

${ }^{\S}$ sushantsk@vecc.gov.in
}

Published by the American Physical Society under the terms of the Creative Commons Attribution 4.0 International license. Further distribution of this work must maintain attribution to the author(s) and the published article's title, journal citation, and DOI. Funded by SCOAP ${ }^{3}$. implies the validity of perturbative analysis of QCD interaction in the high energy limit ${ }^{1}$ [6-14]. The asymptotic freedom also helps us to realize a deconfined state of matter in QCD known as quark-gluon plasma (QGP) at high density and temperature [15].

QGP is a thermal system of deconfined quarks and gluons. It can be created by colliding nuclei at ultrarelativistic energies such as Relativistic Heavy Ion Collider (RHIC) [16] and LHC [17] energies. In our present endeavor, we are interested in the perturbative aspects of QGP where gluon degrees of freedom dominate. Such a state can be created by colliding nuclei at LHC and higher RHIC energies.

The QGP state also provides an opportunity to investigate the nontrivial topological configurations of gauge fields. The nontrivial topological configuration localized in $(3+1)$ dimensions of space-time is known to be instanton. This configuration shows that the Yang-Mills (YM) theory has infinite vacua. These vacua are designated by a parameter $\theta$. The instanton carries a great importance in producing the chiral magnetic effect in QGP when massless quarks are considered. This effect is a combination of electromagnetic and chromomagnetic phenomena [18-21]. The chiral imbalance can help us to investigate the violation of parity $\mathcal{P}$ and $\mathcal{C P}$ symmetries in $\mathrm{QCD}^{2}$ (strong $\mathcal{C P}$ problem).

QGP is considered often with massless gluons. ${ }^{3}$ However, gluons can acquire nonzero masses, i.e., electric and magnetic masses, at finite temperature. The magnetic

\footnotetext{
${ }^{1}$ If the energy of the center of momentum frame of collision is $E$, then here the high energy limit implies $E \gg m$ for any mass $m$ present in the interaction.

${ }^{2}$ Here $\mathcal{C}$ designates charge conjugation operation.

${ }^{3}$ Here "massless gauge field" implies that the gauge field having "bare mass" at zero temperature.
} 
mass is not gauge invariant without resummation [22,23], which is a long-standing problem. The masses carry a great importance in the analysis of QGP [24-26]. Electric mass provides the Debye screening of the static electric field, whereas the nonzero magnetic mass implies the validity of the application of the perturbation technique in the analysis of QGP. Debye mass also plays a pivotal role in the suppression of the effect of large instanton in QGP. On the other hand, it is shown that magnetic mass is absent in massless non-Abelian gauge theory in every loop correction [26], and hence, it is treated in the nonperturbative regime at the length scale $\sim 1 /\left(g^{2} T\right)$, which is much below the scale of the mean free path $\sim 1 /\left(g^{4} T\right)$; here $g(<1)$ is the QCD gauge coupling. It can be shown that the dynamical screening can prevent the infrared singularities in QED plasma, but this would not work for QCD plasma because the massless gluon fields carry color charges.

In this paper, we will construct an effective Lagrangian density by integrating out the hard modes of topologically massive gluons (with momentum $\sim T$ ). This procedure has been followed to obtain a general form of hard thermal loop (HTL) effective action for massless gauge fields [27]. However, we consider an effective action for massive gauge field. The effective action, obtained here for massive gauge fields, will be useful for the computation of the color conductivity and color diffusion constant [28-31] in the perturbative regime. At $T=0$, the massless non-Abelian gauge field has a problem in the description of the local interaction in quantum field theory (QFT) $[32,33]$. Since the Fock space of the non-Abelian gauge field has a positive indefinite metric, the interactions among the massless gluons violate cluster decomposition principle [32,34,35], which is not desirable in a Lorentz-invariant model. On the other hand, the massive gluons can explain the color singlet asymptotic states in physical Hilbert space in QCD [34,36] when color symmetry is not broken spontaneously. However, the presence of mass in the pure non-Abelian gauge theory causes many other problems. For instance, the gauge bosons acquire longitudinal mode, which violates unitarity in the scattering processes at high energy limit. This can be seen in any massive non-Abelian gauge theory, for example, the electroweak sector [37-39]. However, in this sector, these are the Higgs mediated processes, which recover the unitarity of the scattering matrix. But, color symmetry is believed to be an exact symmetry in the strong sector. Hence, the Higgs mechanism and Proca theory cannot be taken into consideration. We can also think of the non-Abelian Stückelberg model, but it was found to be nonrenormalizable [40-44]. The Curci-Ferrari model contains a Proca massive gauge field and it was found to be nonunitary in spite of being renormalizable $[45,46]$. There was also an attempt for the dynamical mass generation of the YM field [47], but that mass vanishes in the high energy limit [48].

The $(3+1)$-dimensional topologically massive model (TMM) contains a topological term: $m B \wedge F=\frac{m}{4} \varepsilon^{\mu \nu \rho \lambda} F_{\mu \nu} B_{\rho \lambda}$
[49], where $B$ is a 2 -form field and $F$ is the field strength of the 1-form gauge field $A$. This is the topological field theory of a Schwarz type [49,50]. This term is a key ingredient for the field theories that are to be independent of metric. For example, in the formulation of quantum gravity, this term is used for the action [51]. In QFT, by considering the kinetic terms of $A$ and $B$ fields, a model can be constructed where observables are related to the local excitations and topological invariants in TMM [50,52]. We observe that the coupling constant $m$ becomes the pole for the gauge field propagator when the $B$ field is integrated out. The spin representation of the $B$ field is different from the $A$ field. Unlike the $A$ field, the massless $B$ field has one degree of freedom, whereas the massive $B$ field behaves like a massive 1-form field in the Lorentz representation [53]. Hence, by integrating out either $A$ or $B$ in the TMM, we obtain an effective field theory for the massive vector bosons. We also see that the TMM is invariant under the vector gauge symmetry of the $B$ field beside the vector gauge symmetry of the YM field. The presence of the infrared cutoff in the non-Abelian generalization of the TMM validates the perturbative analysis in the massive quantum gauge theory. We have recently shown a significant characteristic of the non-Abelian TMM. This is the same behavior of strong coupling at high energy limit (i.e., asymptotic freedom) as what is found in massless YM theory [54]. Hence, this model can be incorporated with the standard model because it does not provide any new degrees of freedom effectively.

The contents of our paper are organized as follows. In Sec. II, we discuss the non-Abelian TMM very briefly. Section III deals with the various vertex rules, propagators of the gauge, and ghost fields present in the TMM. We also show, in this section, how the coupling constant " $m$ " becomes the pole of the complete propagator of the YM field. In the present calculations, the signature of the 4D Minkowski metric $\eta_{\mu \nu}$ is chosen as $\operatorname{diag}(+,-,-,-)$ and $\hbar=k_{B}=1$, where $\hbar$ and $k_{B}$ are the Plank and Boltzmann constants, respectively. In Sec. IV, we estimate the thermal mass for the 1-form massive gauge field at one loop order. In this section, the hard thermal modes of 1-form, 2-form, and ghost fields are integrated out at one loop order and an effective action for soft massive gluons is obtained. Finally, Sec. V has been dedicated to discuss the implication of the results obtained in this work.

\section{II. (3 + 1)-DIMENSIONAL (4D) TOPOLOGICALLY MASSIVE MODEL}

The Lagrangian density of the model is given by [55-57]

$\mathcal{L}=-\frac{1}{4} F_{\mu \nu}^{a} F^{a \mu \nu}+\frac{1}{12} \tilde{H}_{\mu \nu \lambda}^{a} \tilde{H}^{a \mu \nu \lambda}+\frac{m}{4} \varepsilon^{\mu \nu \rho \lambda} B_{\mu \nu}^{a} F_{\rho \lambda}^{a}$,

where the field strengths corresponding the Yang-Mills field $A_{\mu}^{a}$ and the 2-form gauge field $B_{\mu \nu}^{a}$ are, respectively, given by 


$$
F_{\mu \nu}^{a}=\partial_{\mu} A_{\nu}^{a}-\partial_{\nu} A_{\mu}^{a}+g f^{a b c} A_{\mu}^{b} A_{\nu}^{c}
$$

and

$$
\begin{aligned}
\tilde{H}_{\mu \nu \lambda}^{a} & =\left(D_{[\mu} B_{\nu \lambda]}\right)^{a}-g f^{a b c} F_{[\mu \nu}^{b} C_{\lambda]}^{c} \\
& =\partial_{[\mu} B_{\nu \lambda]}^{a}+g f^{a b c} A_{[\mu}^{b} B_{\nu \lambda]}^{c}-g f^{a b c} F_{[\mu \nu}^{b} C_{\lambda]}^{c},
\end{aligned}
$$

where the fields $A_{\mu}^{a}, B_{\mu \nu}^{a}$, and $C_{\mu}^{a}$ are in the adjoint representation of the $S U(N)$ gauge group. Unlike the Abelian model (see Sec. III below), we have an extra vector field $C_{\mu}^{a}$ in this model. It is an auxiliary field [58] that assures the invariance of the Lagrangian density under the following transformations:

$$
A_{\mu}^{a} \rightarrow A_{\mu}^{a} \quad B_{\mu \nu}^{a} \rightarrow B_{\mu \nu}^{a}+\left(D_{[\mu} \theta_{\nu]}\right)^{a}, \quad C_{\mu}^{a} \rightarrow C_{\mu}^{a}+\theta_{\mu}^{a},
$$

where $\theta_{\mu}^{a}$ is a vector field in adjoint representation of $S U(N)$. With inclusion of the Faddeev-Popov ghost fields and Nakanishi-Lautrup fields corresponding to the $A_{\mu}^{a}$ and $B_{\mu \nu}^{a}$ fields, we get the full action that leads to [57]

$$
\begin{aligned}
S= & S_{0}+\int d^{4} x\left[h^{a} f^{a}+\frac{\xi}{2} h^{a} h^{a}-h_{\mu}^{a}\left(f^{a \mu}+\partial^{\mu} n^{a}\right)\right. \\
& -\beta^{a}\left(\partial_{\mu} D^{\mu} \beta^{a}-\partial_{\mu}\left(g f^{a b c} \omega^{b \mu} \omega^{c}\right)\right)-\frac{\eta}{2} h_{\mu}^{a} h^{a \mu} \\
& +\partial_{\mu} \bar{\omega}^{a \mu} \alpha^{a}-\bar{\alpha}^{a} \partial_{\mu} \omega^{a \mu}-\zeta \bar{\alpha}^{a} \alpha^{a}+\bar{\omega}^{a} \partial_{\mu} D^{\mu} \omega^{a} \\
& -\bar{\omega}_{\mu}^{a}\left\{\partial_{\nu}\left(g f^{a b c} B^{b \mu \nu} \omega^{c}\right)+\partial_{\nu}\left(D^{[\mu} \omega^{\nu]}\right)^{a}\right. \\
& \left.\left.+\partial_{\nu}\left(g f^{a b c} F^{b \mu \nu} \theta^{c}\right)\right\}\right],
\end{aligned}
$$

where $S_{0}\left(=\int d^{4} x \mathcal{L}\right)$ is the action corresponding to the Lagrangian density (1) and $f^{a}=\left(\partial^{\mu} A_{\mu}\right)^{a}, f_{\mu}^{a}=\left(\partial^{\nu} B_{\mu \nu}\right)^{a}$. The parameters $\xi, \eta$, and $\zeta$ are the dimensionless gaugefixing parameters. The auxiliary fields $h^{a}$ and $h_{\mu}^{a}$ play the role of Nakanishi-Lautrup-type fields. Here $\omega^{a}$ and $\bar{\omega}^{a}$ are the Faddeev-Popov (FP) ghost and antighost fields (with ghost number +1 and -1 , respectively) corresponding to vector gauge field $A_{\mu}^{a}$. The Lorentz vector ghost fields $\left(\bar{\omega}_{\mu}^{a}\right) \omega_{\mu}^{a}$ [with ghost number $\left.(-1)+1\right)$ are the fermionic (anti-)ghost fields corresponding to tensor field $B_{\mu \nu}^{a}$. The bosonic scalar fields $\left(\bar{\beta}^{a}\right) \beta^{a}$ [with ghost number $(-2)+2$ ) are the (anti-)ghost fields for the fermionic vector (anti-) ghost fields and $n^{a}$ is a bosonic scalar ghost field (with ghost number zero). The latter scalar ghost field is required for the stage-one reducibility of the tensor field. Furthermore, $\alpha^{a}$ and $\bar{\alpha}^{a}$ are the additional Grassmann valued auxiliary fields (having ghost number +1 and -1 ). This model contains a massive non-Abelian gauge field and it was shown to be Becchi-Rouet-Stora-Tyutin (BRST) invariant [59-61]. In [60,61], it is seen that the model is also invariant under the anti-BRST symmetry transformations. It is to be noted that the $\mathcal{C P}$ symmetry is not violated in this model.

\section{VERTEX RULES AND PROPAGATORS OF FIELDS}

The propagators for the $A$ and $B$ fields are found from the Abelian $B \wedge F$ model. The Lagrangian density for the Abelian model is

$$
\mathcal{L}=-\frac{1}{4} F_{\mu \nu} F^{\mu \nu}+\frac{1}{12} H_{\mu \nu \lambda} H^{\mu \nu \lambda}+\frac{m}{4} \varepsilon^{\mu \nu \rho \lambda} B_{\mu \nu} F_{\rho \lambda},
$$

where $F_{\mu \nu}=\partial_{\mu} A_{\nu}-\partial_{\nu} A_{\mu}$ is the field strength of the Abelian gauge field $A_{\mu}, H_{\mu \nu \lambda}=\partial_{\mu} B_{\nu \lambda}+\partial_{\nu} B_{\lambda \mu}+\partial_{\lambda} B_{\mu \nu}$ is the field strength for the tensor field $B_{\mu \nu}$, and $m$ is the coupling constant of the topological term, which has dimension of mass (in natural units $\hbar=c=1$ ). The Lagrangian density is invariant under the following two independent gauge transformations, namely:

$$
\begin{gathered}
A_{\mu} \rightarrow A_{\mu}+\partial_{\mu} \Lambda, \quad B_{\mu \nu} \rightarrow B_{\mu \nu}, \\
A_{\mu} \rightarrow A_{\mu}, \quad B_{\mu \nu} \rightarrow B_{\mu \nu}+\partial_{[\mu} \Lambda_{\nu]},
\end{gathered}
$$

where $\Lambda(x)$ and $\Lambda_{\mu}(x)$ are scalar and vector gauge transformation parameters that vanish at infinity. The EulerLagrange equations of motion derived corresponding to the above Lagrangian are

$$
\begin{aligned}
\partial_{\mu} F^{\mu \nu} & =-\frac{m}{6} \varepsilon^{\nu \mu \lambda \kappa} H_{\mu \lambda \kappa}, \\
\partial_{\mu} H^{\mu \nu \lambda} & =+\frac{m}{2} \varepsilon^{\nu \lambda \kappa \rho} F_{\kappa \rho} .
\end{aligned}
$$

It is interesting to note that one can decouple the above equations for the gauge fields in the following way:

$$
\left(\square+m^{2}\right) F_{\mu \nu}=0, \quad\left(\square+m^{2}\right) H_{\mu \nu \lambda}=0,
$$

which shows the well-known Klein-Gordon equations for the massive fields $A_{\mu}$ and $B_{\mu \nu}$.

We will consider the loop calculation, which requires the propagators of $A_{\mu}$ and $B_{\mu \nu}$ fields. To achieve this, we introduce the gauge-fixing terms in the Lagrangian density given in Eq. (6) as

$$
\mathcal{L}_{g f}=-\frac{1}{2 \xi}\left(\partial_{\mu} A^{\mu}\right)^{2}+\frac{1}{2 \eta}\left(\partial_{\mu} B^{\mu \nu}\right)^{2},
$$

where $\xi$ and $\eta$ are the gauge-fixing parameters. The topological term is also quadratic in nature, containing both $A_{\mu}$ and $B_{\mu \nu}$ fields. To calculate the propagator of the fields, we should take all the quadratic terms in the Lagrangian density, excluding the $B \wedge F$ term. The propagators of $A_{\mu}$ and $B_{\rho \lambda}$ fields are given by 


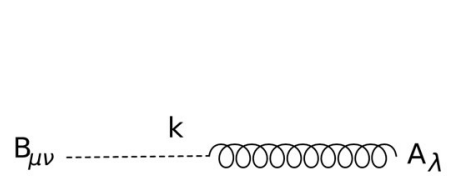

(a)

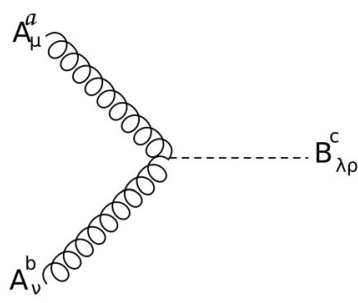

(b)

FIG. 1. (a) $B A$ vertex and (b) $A B B$ vertex from $B \wedge F$ term.

$$
\begin{gathered}
i \Delta_{\mu \nu}=-\frac{i}{k^{2}}\left(\eta^{\mu \nu}-(1-\xi) \frac{k^{\mu} k^{\nu}}{k^{2}}\right) \\
i \Delta_{\mu \nu, \rho \lambda}=\frac{i}{k^{2}}\left(\eta_{\mu[\rho} \eta_{\lambda] \nu}-(1-\eta) \frac{k_{\mu} k_{[\lambda} \eta_{\rho] \nu}-k_{\nu} k_{[\lambda} \eta_{\rho] \mu}}{k^{2}}\right) .
\end{gathered}
$$

The vertex for the interaction term containing these fields is given by

$$
i V_{\mu \nu, \lambda}=-m \varepsilon_{\mu \nu \lambda \rho} k^{\rho}
$$

which is shown in Fig. 1. The complete propagator for the vector field $A_{\mu}$ can be obtained by taking an infinite number of insertions of the $B A$ vertex and the $B$ propagator [cf. Eq. (13)]. This process is shown in Fig. 2 and the sum of diagrams can be written as the infinite sum, as shown in Fig. 2. Thus, the complete propagator for massive vector bosons is given by

$$
\begin{aligned}
i D_{\mu \nu} & =i \Delta_{\mu \nu}+i \Delta_{\mu \mu^{\prime}} \frac{1}{2} i V^{\sigma \rho, \mu^{\prime}} i \Delta_{\sigma \rho, \sigma^{\prime} \rho^{\prime}} \frac{1}{2} i V^{\sigma^{\prime} \rho^{\prime}, \nu^{\prime}} i \Delta_{\nu^{\prime} \nu}+\cdots \\
& =-i\left[\frac{\eta_{\mu \nu}-(1-\xi) \frac{k_{\mu} k_{\nu}}{k^{2}}}{\left(k^{2}-m^{2}\right)}-\xi m^{2} \frac{k_{\mu} k_{\nu}}{k^{4}\left(k^{2}-m^{2}\right)}\right],
\end{aligned}
$$

where $m$, appearing as a pole of the propagators, clearly represents the mass of vector gauge bosons. The factors of $\frac{1}{2}$ compensate for double counting due to the antisymmetrization of the indices. Similarly, for the tensor field $B$, we have the following propagator:

$i D_{\mu \nu, \rho \lambda}=\left[\frac{\eta_{\mu[\rho} \eta_{\lambda] \nu}+(1-\eta) \frac{k_{[\mu} k_{\lambda} \eta_{\rho / \nu}}{k^{2}}}{k^{2}-m^{2}}+\eta m^{2} \frac{k_{[\mu} k_{[\lambda} \eta_{\rho] \nu]}}{k^{4}\left(k^{2}-m^{2}\right)}\right]$.

The kinetic term of the YM field [cf. Eq. (5)] provides the derivative trilinear and quartic couplings. The interaction part of the kinetic term of YM field is

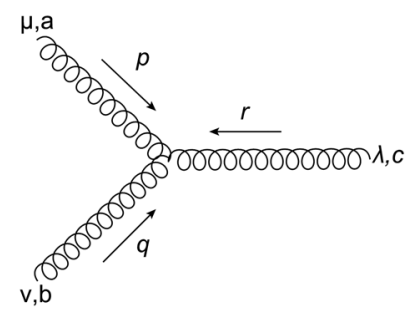

(a)

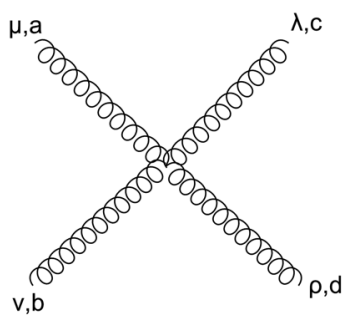

(b)
FIG. 3. (a) $A A A$ trilinear vertex and (b) $A A A A$ quartic vertex.

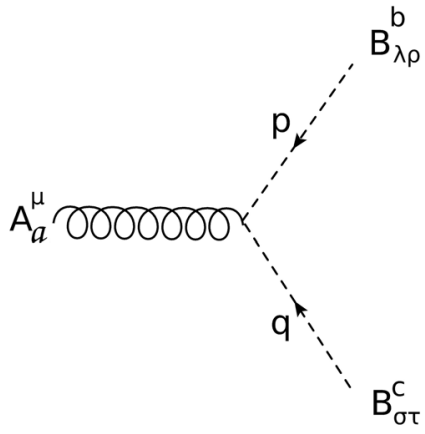

(a)

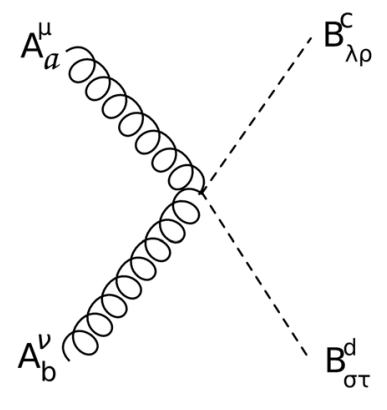

(b)
FIG. 4. (a) $A B B$ and (b) $A A B B$ vertices.

$$
\mathcal{L}_{\text {int }}=\frac{1}{4} g f^{b c a} A^{\mu b} A^{\nu c}\left(\partial_{[\mu} A_{\nu]}^{a}-2 g f^{d e a} A_{\mu}^{d} A_{\nu}^{e}\right) .
$$

The vertex rules corresponding to these couplings are as follows:

$$
\begin{gathered}
V_{\mu \nu \lambda}^{a b c}=-g f^{a b c}\left[(q-r)_{\mu} \eta_{\nu \lambda}+(r-p)_{\nu} \eta_{\lambda \mu}\right. \\
\left.+(p-q)_{\lambda} \eta_{\mu \nu}\right], \\
V_{\mu \nu \lambda \rho}^{a b c d}=-i g^{2}\left[f^{a b e} f^{c d e} \eta_{\mu[\lambda} \eta_{\rho] \nu}+f^{a c e} f^{b d e} \eta_{\mu[\nu} \eta_{\rho] \lambda}\right. \\
\left.+f^{a d e} f^{b c e} \eta_{\mu[\nu} \eta_{\lambda] \rho}\right],
\end{gathered}
$$

where $f$ 's are the structure constants of the $S U(N)$ group, which are totally antisymmetric in their indices. The momenta of the particles at the trilinear vertex is shown in Fig. 3(a). The topological term also provides a trilinear coupling $A B B$ with vertex term

$$
i V_{\mu, \nu, \lambda \rho}^{a b c}=-i g m f^{b c a} \varepsilon_{\mu \nu \lambda \rho} .
$$

To proceed further, we require the propagators of vector ghost fields $\omega^{\mu}$ and $\bar{\omega}^{\mu}$, the ghost fields of the vector ghost fields $\beta$ and $\bar{\beta}$, and the ghost fields $\omega$ and $\bar{\omega}$ corresponding

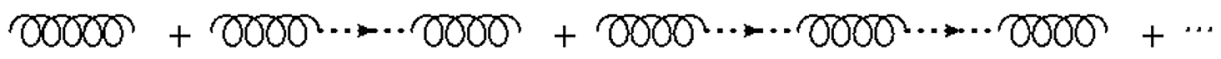

FIG. 2. Massive $A$ propagator by summing over $B$ insertions. 
to the 1-form gauge field, which appeared in Eq. (5). The vertices for trilinear and quartic couplings $A B B$ and $A A B B$, shown in Fig. 4, are

$$
\begin{aligned}
i V_{\mu, \lambda \rho, \sigma \tau}^{a b c}= & g f^{a b c}\left[(p-q)_{\mu} \eta_{\lambda[\sigma} \eta_{\tau] \rho}+p_{[\sigma} \eta_{\tau][\lambda} \eta_{\rho] \mu}\right. \\
& \left.-q_{[\lambda} \eta_{\rho][\sigma} \eta_{\tau] \mu}\right], \\
i V_{\mu, \nu, \lambda \rho, \sigma \tau}^{a b c d}= & i g^{2}\left[f^{a c e} f^{b d e}\left(\eta_{\mu \nu} \eta_{\lambda[\sigma} \eta_{\tau] \rho}+\eta_{\mu[\sigma} \eta_{\tau][\lambda} \eta_{\rho] \nu}\right)\right. \\
& \left.+f^{a d e} f^{b c e}\left(\eta_{\mu \nu} \eta_{\lambda[\sigma} \eta_{\tau] \rho}+\eta_{\mu[\lambda[} \eta_{\rho][\sigma} \eta_{\tau] \nu}\right)\right] .
\end{aligned}
$$

\section{a,a $\aleph^{W_{v, b}}$ \\ Nonvo}

Apart from the usual trilinear coupling among FaddeevPopov (FP) ghost and YM fields, we can also see the action contains another trilinear coupling among vector ghosts and YM fields, which is given by

$$
\mathcal{L}_{\text {int }}^{v e c-g h-A}=-g f^{a b c} \partial^{\nu} \bar{\omega}^{\mu a} A_{[\mu}^{b} \omega_{\nu]}^{c} .
$$

The vertex factor corresponding to this Lagrangian density is given by

$$
i V_{\mu \nu \lambda}^{a b c}=-g f^{a b c}\left(p_{\nu} \eta_{\mu \lambda}-p_{\mu} \eta_{\nu \lambda}\right) .
$$

In the derivation of the above rules, all four momentums are taken as incoming toward the vertex, as shown in Fig. 5(a). There is also a trilinear coupling among the YM and ghost of the vector ghost fields. The trilinear vertex is shown in Fig. 5(b). The coupling is given by the following Lagrangian density:

$$
\mathcal{L}_{\text {int }}^{A \beta \bar{\beta}}=-g f^{b c a} A_{\mu}^{b} \partial^{\mu} \bar{\beta}^{a} \beta^{c} .
$$

The propagator of the vector ghost field from the Lagrangian density

$\mathcal{L}=-\partial_{\mu} \bar{\omega}_{\nu}^{a}\left(\partial^{\mu} \omega^{\nu a}-\partial^{\nu} \omega^{\mu a}\right)-\frac{1}{\tilde{\xi}}\left(\partial_{\mu} \omega^{\mu a}\right)\left(\partial_{\nu} \bar{\omega}^{\nu a}\right)$,

at the gauge $\tilde{\xi}=1$ can be obtained by integrating out $\alpha$ and $\bar{\alpha}$ from the action in Eq. (5) as

$i \Delta_{\omega \bar{\omega}, a b}=-\frac{i}{p^{2}}\left[\eta^{\mu \nu}-(1-\tilde{\xi}) \frac{p^{\mu} p^{\nu}}{p^{2}}\right] \delta_{a b}$,

$$
i \Delta_{\bar{\beta}, \beta, a b}=\frac{i}{p^{2}} \delta_{a b} .
$$

Since, the coupling in Eq. (26) also contains the derivative of fields, the corresponding vertex will be momentum dependent. This trilinear coupling is same as the trilinear coupling among the YM field and its FP ghosts with the vertex term and it is given by

$$
i V_{a b c}^{\mu}=-g f_{a b c} p^{\mu}
$$

\section{ONE LOOP CORRECTION}

Using the vertices and the propagators derived above, we calculate the one loop correction of the soft modes of massive gluons with the scope of the Feynman-'t Hooft gauge: $\xi=\eta=\tilde{\xi}=1$ in 4D Euclidean space where we replace the Minkowski metric $\eta^{\mu \nu}$ by the Euclidean metric $\delta^{\mu \nu}$. The amplitude of the one loop diagram can be written generically as

$$
\Pi_{\mu \nu}^{a b}=\frac{g^{2} N_{C}}{n} \delta^{a b} \underbrace{}_{\mathbf{p}} \frac{\delta_{\mu \nu}\left(a_{1} p^{2}+a_{2} k^{2}+a_{3} m^{2}\right)+a_{4} k_{\mu} k_{\nu}+a_{4}\left(p_{\mu} k_{\nu}+p_{\nu} k_{\mu}\right)+a_{5} p_{\mu} p_{\nu}}{\left(p^{2}-m^{2}\right)\left\{(k-p)^{2}-m^{2}\right\}},
$$

where $\mathbb{Z}_{\mathbf{p}} \equiv \mathbb{Z}_{\vec{p}}=\sum_{p_{0 n}} T \int_{\mathbf{p}}, \int_{\mathbf{p}}=\int \frac{d^{d} \mathbf{p}}{(2 \pi)^{d}}$. We assume that the external legs carry soft momenta $\sim g T$. We take the Matsubara sum over the temporal component $p_{\text {on }}$ of the four momentum $p_{\mu}$ and integrate over spatial component ${ }^{4}$

\footnotetext{
${ }^{4}$ We use here slightly unconventional notation, since $p_{\mu}$ and $k_{\mu}$ designate the four momenta. $P$ and $K$ stand for the magnitude of three momenta. The spatial components of momentum are denoted as $p_{i}$ 's (or $k_{i}$ 's) $(i=1,2,3)$.
}

$\mathbf{p} \equiv\left\{p_{i}\right\}$. To carry out this, we approximate the energies $E_{1}$ and $E_{2}$ of the external legs as follows:

$$
\begin{gathered}
E_{1}=\sqrt{\mathbf{p}^{2}+m^{2}} \approx P+\frac{m^{2}}{2 P}+\cdots, \\
E_{2}=\sqrt{(\mathbf{p}-\mathbf{k})^{2}+m^{2}} \approx P-\mathbf{k} \cdot \mathbf{v}+\frac{m^{2}}{2 P},
\end{gathered}
$$

where $|\mathbf{p}|=P$ and $v_{i}=\frac{p_{i}}{P}$. Now we consider 


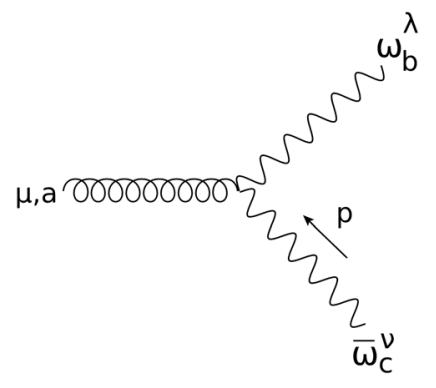

(a)

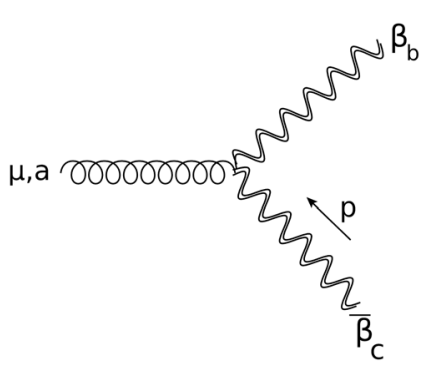

(b)
FIG. 5. (a) Trilinear vertex among $A_{\mu}, \bar{\omega}_{\nu}$, and $\omega_{\lambda}$. (b) Trilinear vertex among $A_{\mu}, \beta$, and $\bar{\beta}$. Wavy line designates the vector ghosts in (a) and ghosts of the vector ghost fields are represented as a double wavy line in (b).

$$
\mathcal{G}=T \sum_{p_{0 n}} \frac{1}{\left(p_{0 n}^{2}+E_{1}^{2}\right)\left\{(k-p)_{0 n}^{2}-m^{2}\right\}}
$$

which, summing over $p_{0 n}$, results in

$$
\begin{aligned}
\mathcal{G}= & \frac{1}{4 E_{1} E_{2}}\left[\frac{1}{i k_{\mathrm{on}}-E_{1}-E_{2}}\left(-n_{B}\left(E_{1}\right)-n_{B}\left(E_{2}\right)-1\right)\right. \\
& +\frac{1}{i k_{\mathrm{on}}+E_{2}-E_{1}}\left(n_{B}\left(E_{1}\right)-n_{B}\left(E_{2}\right)\right) \\
& +\frac{1}{i k_{\mathrm{on}}+E_{1}-E_{2}}\left(n_{B}\left(E_{2}\right)-n_{B}\left(E_{1}\right)\right) \\
& \left.+\frac{1}{i k_{\mathrm{on}}+E_{2}+E_{1}}\left(1+n_{B}\left(E_{1}\right)+n_{B}\left(E_{2}\right)\right)\right] .
\end{aligned}
$$

The quadratic terms $\mathcal{O}\left(p^{2}\right)$ are neglected from the numerator because of the assumption $p \sim g T(g \ll T)$. Using Eqs. (29) and (30), the above expression of $\mathcal{G}$ can be approximated as

$$
\tilde{\mathcal{G}} \approx\left[-\frac{1}{2 P}\left\{-2 n_{B}(P)\right\}+\Sigma(\mathbf{p})+\frac{1}{2 P}\left\{2 n_{B}(P)\right\}\right],
$$

where

$$
\begin{aligned}
\Sigma(\mathbf{p})= & \left(\frac{1}{i k_{\mathrm{on}}+E_{2}-E_{1}}\left(n_{B}\left(E_{1}\right)-n_{B}\left(E_{2}\right)\right)\right. \\
& \left.+\frac{1}{i k_{\mathrm{on}}+E_{1}-E_{2}}\left(n_{B}\left(E_{2}\right)-n_{B}\left(E_{1}\right)\right)\right) .
\end{aligned}
$$

The denominator in Eq. (28) contains identical propagators of bosons. Hence, the term $\left(p_{\mu} k_{\nu}+p_{\nu} k_{\mu}\right)$ can be simplified by renaming the variable $p \rightarrow k-p$, and considering one half of this term as

$$
\begin{aligned}
p_{\mu} k_{\nu}+p_{\nu} k_{\mu} \rightarrow & \frac{1}{2}\left[p_{\mu} k_{\nu}+p_{\nu} k_{\mu}+(k-p)_{\mu} k_{\nu}\right. \\
& \left.+(k-p)_{\nu} k_{\mu}\right]=k_{\mu} k_{\nu} .
\end{aligned}
$$

After rearranging the terms in the numerator of the integrand in Eq. (28) and neglecting the term $\sim \mathcal{O}\left(K^{2}\right)$, the spatial part reads as

$$
\begin{aligned}
N_{i j}^{a b}= & \frac{g^{2} N_{C} \delta^{a b}}{n}\left[\delta_{i j}\left(a_{1} p^{2}+a_{3} m^{2}\right)+a_{5} p_{i} p_{j}\right] \\
= & \frac{g^{2} N_{C} \delta^{a b}}{n}\left[\delta_{i j}\left(a_{1}\left(p^{2}-m^{2}\right)+a_{1} m^{2}+a_{3} m^{2}\right)\right. \\
& \left.+a_{5} p_{i} p_{j}\right] \\
= & \frac{g^{2} N_{C} \delta^{a b}}{n}\left[\delta_{i j}\left(a_{1}\left(p^{2}-m^{2}\right)+\left(a_{1}+a_{3}\right) m^{2}\right)\right. \\
& \left.+a_{5} p_{i} p_{j}\right] .
\end{aligned}
$$

From the above expression, it is clear that the presence of the term $m^{2} \delta_{i j}$ will provide the magnetic mass of gluons. In constructing an effective field theory, we neglect the quadratic term of $\mathcal{O}\left(K^{2}\right)$ from the above expression. Substituting $\mathcal{G}$ in Eq. (33), the spatial part of the $\Pi_{\mu \nu}$, which, after changing the variable $\mathbf{v} \rightarrow-\mathbf{v}$, reads

$$
\begin{aligned}
\Pi_{i j}^{a b} \approx & \frac{g^{2} N_{C}}{n} \delta^{a b} \int_{\mathbf{p}}\left[a_{1} \frac{n_{B}(P)}{P} \delta_{i j}\right. \\
& \left.+\left(\tilde{A} m^{2} \delta_{i j}+a_{5} p_{i} p_{j}\right)\left\{\frac{n_{B}(P)}{2 P^{3}}+\Sigma(\mathbf{p})\right\}\right],
\end{aligned}
$$

where $\quad \tilde{A}=\left(a_{1}+a_{3}\right), \quad \int_{p}=c(d) \int p^{d-1} d p, \quad$ and $c(d)=\frac{2}{(4 \pi)^{d / 2} \Gamma(d / 2)}$. The angular integration goes over directions of $v_{i} \equiv \frac{p_{i}}{P}$ and is normalized to unity as

$$
\int d \Omega_{v}=1
$$

and using the rotational invariance, we get

$$
\int d \Omega_{v} v_{i} v_{j}=\frac{1}{d} \delta_{i j}
$$

Thus, we are going to construct the effective field theory in the energy scale $E=\sqrt{P^{2}+m^{2}}$, where $m \ll E \leq T$. Using Eq. (39) and the following identity for $d=3$ :

$$
\int_{P} n_{B}^{\prime}(P)=-(d-1) \int_{P} \frac{1}{P} n_{B}(P)
$$

$\Pi_{\mu \nu}^{a b}$ can be expressed as 


$$
\begin{aligned}
\Pi_{i j}^{a b}= & \delta^{a b} \frac{g^{2} N_{c}}{n}\left[\int _ { \mathbf { p } } \frac { n _ { B } ( P ) } { P } \left\{\left(a_{1}+\frac{a_{5}}{2 d}-a_{5} \frac{(d-1)}{2 d}\right) \delta_{i j}\right.\right. \\
& \left.-\frac{(d-1)}{2} a_{5} \int d \Omega_{v} \frac{v_{i} v_{j} k_{0 n}}{i k_{0 n}-\mathbf{k} \cdot \mathbf{v}}\right\} \\
& \left.+\tilde{A} m^{2} \delta_{i j} \int d \Omega_{v} \Sigma(\mathbf{p})\right] .
\end{aligned}
$$

The last term of the above integrand can be written as

$$
m^{2} \int d \Omega_{v} \Sigma(\mathbf{p})=m^{2} I
$$

where ${ }^{5}$

$$
\begin{aligned}
I= & 2 \sqrt{2} \frac{1}{(2 \pi)^{2} K} \sum_{s=1}^{\infty} m K_{1}(\sqrt{2} s m \beta) \operatorname{Re} \\
& \times\left[e^{i s \beta k_{0 n}} E_{1}\left(s \beta\left\{K+i k_{0 n}\right\}\right)-E_{1}\left(s \beta\left\{-K+i k_{0 n}\right\}\right)\right] .
\end{aligned}
$$

With the help of Eq. (30), we can now reexpress Eq. (41) as

$$
\begin{aligned}
\Pi_{i j}= & \frac{g^{2} N_{C}}{n}\left[\left\{\tilde{B} \frac{T^{2}}{12}+\tilde{A} m^{2} I\right\} \delta_{i j}\right. \\
& \left.+C \frac{T^{2}}{12}\left(\mathcal{P}_{i j}^{T} \Pi_{T}(K)+\mathcal{P}_{i j}^{E} \Pi_{E}(k)\right)\right],
\end{aligned}
$$

where in $d$ spatial dimensions, $\tilde{B}=\left(a_{1}+\frac{a_{5}}{2 d}-\frac{a_{5}(d-1)}{2 d}\right)$ and $C=-\frac{(d-1)}{2} a_{5}$. The factor $\frac{T^{2}}{12}$ appears from the integration $\int_{P} \frac{1}{P} n_{B}(P)$, where $\int_{P} \equiv V(d) \int_{0}^{\infty} p^{d-1} d p$ and $V(d)=\frac{2}{(4 \pi)^{\frac{d}{2}} \Gamma\left(\frac{d}{2}\right)}$. The gauge indices in the above calculations have been suppressed and the coefficients of the projection operators are found as

$$
\mathcal{P}_{\mu \nu}^{T}(k) \equiv \delta_{\mu i} \delta_{\nu j} P_{i j}^{T}(k),
$$

$$
\mathcal{P}_{\mu \nu}^{E} \equiv \delta_{\mu \nu}-\frac{k_{\mu} k_{\nu}}{k^{2}}-\mathcal{P}_{\mu \nu}^{T}(k),
$$

where $P_{i j}^{T}(k)=\delta_{i j}-k_{i} k_{j} / K^{2}$ and in the three dimensions [62]

$\Pi_{T}(K)=\frac{1}{2}\left[\frac{\left(i k_{0 n}\right)^{2}}{K^{2}}+\frac{i k_{0 n}}{2 K}\left\{1-\frac{\left(i k_{0 n}\right)^{2}}{K^{2}}\right\} \ln \frac{i k_{0 n}+K}{i k_{0 n}-K}\right]$,

$$
\Pi_{E}(K)=\left[1-\frac{\left(i k_{0 n}\right)^{2}}{K^{2}}\right]\left[1-\frac{i k_{0 n}}{2 K} \ln \frac{i k_{0 n}+K}{i k_{0 n}-K}\right] .
$$

Now we can write the effective Lagrangian density as

$$
\begin{aligned}
\mathcal{L}_{\text {eff }}= & -\frac{1}{4} F_{a}^{\mu \nu} F_{\mu \nu}^{a}+\int_{K} \tilde{m}^{2}(K) A^{\mu}(K) A_{\mu}(-K) \\
& +m_{E}^{2} \int d \Omega_{v}\left(\frac{1}{\mathcal{V} \cdot D} \mathcal{V}^{\alpha} F_{\alpha \mu}^{a}\right)\left(\frac{1}{\mathcal{V} \cdot D} \mathcal{V}^{\beta} F_{a \beta}^{\mu}\right),
\end{aligned}
$$

where $\quad \tilde{m}^{2}=\frac{g^{2} N_{c}}{n}\left[\tilde{B} \frac{T^{2}}{12}+\tilde{A} m^{2} I\right], \quad m_{E}^{2} \approx g^{2} C \frac{N_{c}}{n} \frac{T^{2}}{12}, \quad$ and $\mathcal{V}^{\alpha} \equiv(1, \mathbf{v})$. We have obtained a generic form of the Debye mass and observed how the bare mass of gluon contributes in the construction of effective action.

Now we proceed to consider the relevant contributions to the effective Lagrangian from various loop diagrams of the topologically massive model. The generic form of the loop integration is given as

$$
\Pi_{i j}=m^{2} \bigvee_{\mathbf{k}} \frac{\left(\tilde{A} \delta_{i j} k^{2}+\tilde{B} k_{i} k_{j}\right)}{k^{2}\left\{(p-k)^{2}-m^{2}\right\}\left(k^{2}-m^{2}\right)},
$$

which could be written as

$$
\Pi_{i j}=m^{2} \int_{\mathbf{k}}\left(\tilde{A} \delta_{i j} k^{2}+\tilde{B} k_{i} k_{j}\right) \mathcal{G}\left(E_{1}, E_{2}, E_{3}, k\right),
$$

and

$$
\mathcal{G}\left(E_{1}, E_{2}, E_{3}, k\right)=\sum_{n} \frac{1}{\left(k_{0 n}^{2}+E_{1}^{2}\right)\left\{(p-k)_{0 n}^{2}+E_{2}^{2}\right\}\left(k_{0 n}^{2}+E_{3}^{2}\right)}=\frac{1}{E_{3}^{2}-E_{1}^{2}}\left[\mathcal{G}\left(E_{1}, E_{2}, k\right)-\mathcal{G}\left(E_{2}, E_{3}, k\right)\right]
$$

where

$$
\begin{aligned}
\mathcal{G}\left(E_{1}, E_{2}, k\right)= & T \sum_{p_{0 n}} \frac{1}{\left(p_{0 n}^{2}+E_{1}^{2}\right)\left\{r_{0 n}^{2}+E_{2}^{2}\right\}} \\
= & \frac{1}{4 E_{1} E_{2}}\left[\frac{1}{i k_{\mathrm{on}}-E_{1}-E_{2}}\left(-n_{B}\left(E_{1}\right)-n_{B}\left(E_{2}\right)-1\right)+\frac{1}{i k_{\mathrm{on}}+E_{2}-E_{1}}\left(n_{B}\left(E_{1}\right)-n_{B}\left(E_{2}\right)\right)\right. \\
& \left.+\frac{1}{i k_{\mathrm{on}}+E_{1}-E_{2}}\left(n_{B}\left(E_{2}\right)-n_{B}\left(E_{1}\right)\right)+\frac{1}{i k_{\mathrm{on}}+E_{2}+E_{1}}\left(1+n_{B}\left(E_{1}\right)+n_{B}\left(E_{2}\right)\right)\right] .
\end{aligned}
$$

\footnotetext{
${ }^{5}$ See the detail in Appendix A.
} 
Thus, from Eqs. (52) and (53), we get

$$
\begin{aligned}
\mathcal{G}= & \frac{1}{m^{2}}\left[\frac { 1 } { 4 E _ { 1 } E _ { 2 } } \left\{\frac{1}{i k_{\mathrm{on}}-E_{1}-E_{2}}\left(-n_{B}\left(E_{1}\right)-n_{B}\left(E_{2}\right)-1\right)\right.\right. \\
& +\frac{1}{i k_{\mathrm{on}}+E_{2}-E_{1}}\left(n_{B}\left(E_{1}\right)-n_{B}\left(E_{2}\right)\right)+\frac{1}{i k_{\mathrm{on}}+E_{1}-E_{2}}\left(n_{B}\left(E_{2}\right)-n_{B}\left(E_{1}\right)\right) \\
& \left.+\frac{1}{i k_{\mathrm{on}}+E_{2}+E_{1}}\left(1+n_{B}\left(E_{1}\right)+n_{B}\left(E_{2}\right)\right)\right\} \\
& -\frac{1}{4 E_{3} E_{2}}\left\{\frac{1}{i k_{\mathrm{on}}-E_{2}-E_{3}}\left(-n_{B}\left(E_{2}\right)-n_{B}\left(E_{3}-\mu\right)-1\right)\right. \\
& +\frac{1}{i k_{\mathrm{on}}+E_{2}-E_{3}}\left(n_{B}\left(E_{3}\right)-n_{B}\left(E_{2}-\mu\right)\right)+\frac{1}{i k_{\mathrm{on}}+E_{3}-E_{2}}\left(n_{B}\left(E_{2}\right)-n_{B}\left(E_{1}\right)\right) \\
& \left.\left.+\frac{1}{i k_{\mathrm{on}}+E_{2}+E_{3}}\left(1+n_{B}\left(E_{3}\right)+n_{B}\left(E_{2}\right)\right)\right\}\right]
\end{aligned}
$$

where $\frac{1}{m^{2}}$ originates from $E_{3}^{2}-E_{1}^{2}=k_{0 n}^{2}+m^{2}-k_{0 n}^{2}=m^{2}$. Taking the HTL approximation, we can write the above expression in the following form:

$$
\mathcal{G} \approx \frac{n_{B}\left(E_{1}\right)-n_{B}\left(E_{3}\right)}{4 K^{2} m^{2}}\left[\frac{1}{i p_{0 n}-\mathbf{p} \cdot \mathbf{v}}-\frac{1}{i p_{0 n}+\mathbf{p} \cdot \mathbf{v}}\right] .
$$

The task will become simple with the observation that the diagrams in Fig. 6 are to be neglected in HTL approximation. In this approximation $m \ll K$, then $n_{B}\left(E_{1}\right) \approx n_{B}\left(E_{3}\right)$ at leading order. Hence, the contribution to the quantum corrections from the diagrams in Fig. 6(a) and $6(\mathrm{~b})$ is given by $\Pi_{i j}^{6 a, 6 b} \approx 0$. A similar conclusion can be drawn for the contribution from Fig. 6(c), which contains four propagators. Therefore, the $\Pi_{i j}^{6 c}$ have the Matsubara sum as

$$
\mathcal{G}\left(E_{1}, E_{2}, E_{3}, E_{4}, k\right)=\bigcup_{\mathbf{K}} \frac{1}{\left(k_{0 n}^{2}+E_{1}^{2}\right)\left\{(p-k)_{0 n}^{2}+E_{2}^{2}\right\}\left(k_{0 n}^{2}+E_{3}^{2}\right)\left\{(p-k)_{0 n}^{2}+E_{4}^{2}\right\}} .
$$

After some algebraic manipulation and HTL approximation, the above expression becomes

$$
\mathcal{G}\left(E_{1}, E_{2}, E_{3}, E_{4}, k\right) \approx \frac{\left(n_{B}\left(E_{3}\right)-n_{B}\left(E_{1}\right)\right)}{4 K^{2} m^{4}}\left[\left(\frac{1}{i p_{n}-\mathbf{p} \cdot \mathbf{v}}-\frac{1}{i p_{n}+\mathbf{p} \cdot \mathbf{v}}\right)+\left(\frac{1}{i p_{n}+\mathbf{p} \cdot \mathbf{v}}-\frac{1}{i p_{n}-\mathbf{p} \cdot \mathbf{v}}\right)\right],
$$

which shows that $\Pi_{i j}^{6 c}$ does not contribute too.

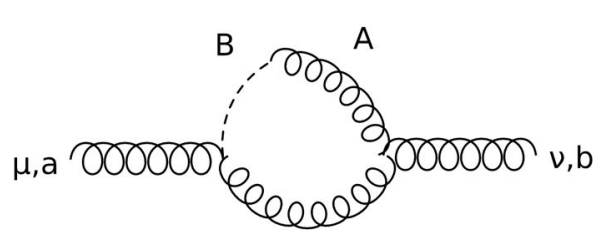

(a)

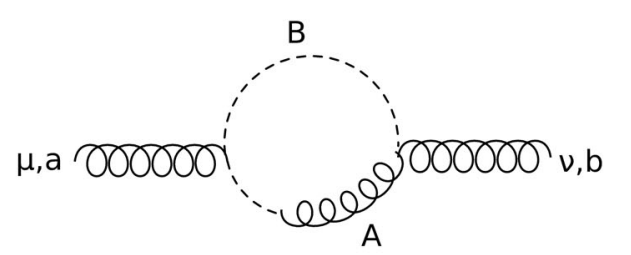

(b)

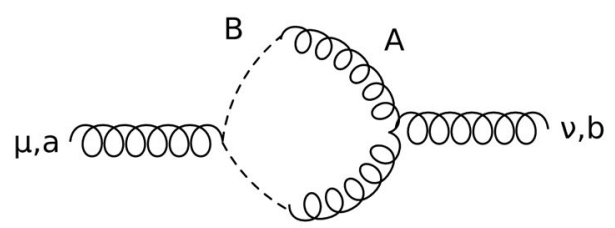

(c)

FIG. 6. Loop diagrams formed by (a) $A A A$ and $A A B$, (b) $A B B$ and $A A B$, (c) $A A A$ and $A B B$ couplings that do not contribute in the HTL approximation. 


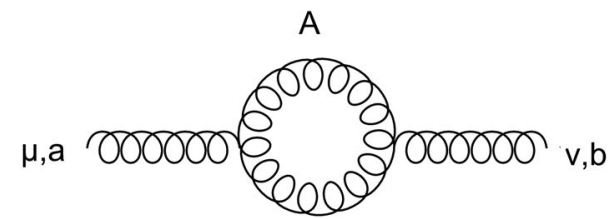

(a)

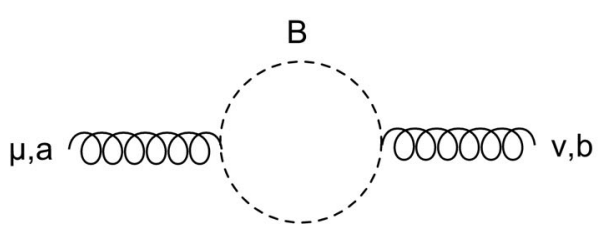

(c)

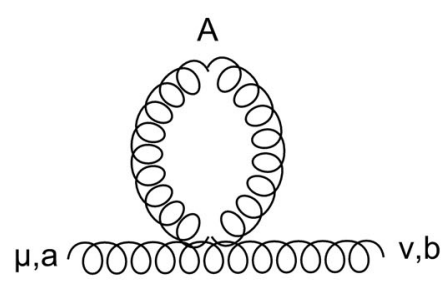

(b)

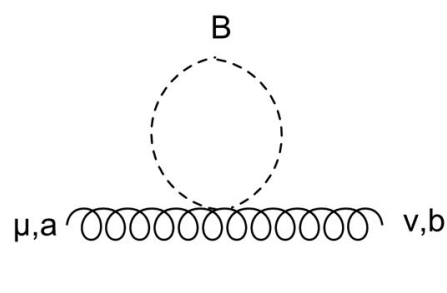

(d)

FIG. 7. Loop diagrams formed by (a) $A A A$, (b) $A A A A$, (c) $A B B$, and (d) $A A B B$ couplings.

Only relevant loop diagrams with nonzero contribution, constructed from $A$ and $B$ fields, are shown in Fig. 7. The rest of the diagrams are from the ghost sectors, where loops are constructed by the FP ghost of the YM field $\omega$ and $\bar{\omega}$, vector ghost $\omega^{\mu}$ and $\bar{\omega}^{\mu}$, and ghost of the vector ghost $\beta$ and $\bar{\beta}$ corresponding to tensor field $B_{\mu \nu}$.

We easily reach at the conclusion from Fig. 7(a) that the term $m^{2} / K^{4}$ in the propagator of the massive YM field is irrelevant under the approximation considered here, i.e., when $K$ is hard. Instead of the propagator behaving as $\sim 1 / k^{2}$, now we have to consider $1 /\left(k^{2}-m^{2}\right)$. On the other hand, the vertex rule of trilinear coupling among the massive gluon fields is same as that of the massless YM field. This makes the calculation easier. We have also noticed that the loop amplitude from Fig. 7(a) in the HTL approximation at the leading order is same as that of the massless YM case because of the structure of the propagator of the massive YM field. On the other hand, the trilinear vertex rule among the massive YM field and its massless ghosts is same as that of the massless YM theory. These similarities imply that the thermal loop amplitude for Fig. 9(a) is the same as found in that of massless YM theory. The contributions from Figs. 7(a) and $7(b)$ are

$$
\begin{aligned}
\Pi_{\mu \nu}^{7 a} & =\frac{g^{2} N_{c}}{2} \underbrace{}_{\vec{k}} \frac{-\delta_{\mu \nu}\left[5 p^{2}-2 p \cdot k+2 k^{2}\right]+(d+4) p_{\mu} p_{\nu}-(4 d-2) k_{\mu} k_{\nu}}{\left(k^{2}-m^{2}\right)\left[(p-k)^{2}-m^{2}\right]}, \\
\Pi_{\mu \nu}^{7 b} & =-\frac{1}{2} g^{2} N_{c} \delta_{\mu \nu} \sum_{\vec{k}} \frac{2 d}{\left(k^{2}-m^{2}\right)} .
\end{aligned}
$$

Neglecting the terms $\sim \mathcal{O}\left(p^{2}\right)$ in the numerator of Eq. (58), we get the spatial part as

$$
\begin{aligned}
\Pi_{i j}^{7 a} & \approx \frac{g^{2} N_{c}}{2} \underbrace{}_{\vec{k}} \frac{-\delta_{i j}\left[-2 p \cdot k+2 k^{2}\right]-(4 d-2) k_{i} k_{j}}{\left(k^{2}-m^{2}\right)\left[(p-k)^{2}-m^{2}\right]} \\
& =-g^{2} N_{c} \underbrace{}_{\vec{k}} \frac{\delta_{i j}\left[-p \cdot k+k^{2}\right]+(2 d-1) k_{i} k_{j}}{\left(k^{2}-m^{2}\right)\left[(p-k)^{2}-m^{2}\right]} .
\end{aligned}
$$

Comparing Eq. (59) with Eq. (44), we find that $a_{1}=-1$, $a_{3}=0, a_{5}=-5$, and $n=1$ for $d=3$. Next, we consider the diagram in Fig. 7(b), which provides the spatial part of the loop amplitude as

$$
\begin{aligned}
& \Pi_{i j}^{7 b}=-g^{2} N_{c} \delta_{i j} \oiint_{\vec{k}} \frac{d}{\left(k^{2}-m^{2}\right)} \\
& \approx-g^{2} d N_{c} \delta_{i j} \int_{\vec{k}} \frac{1}{2 K}\left(1+n_{B}(K)\right) .
\end{aligned}
$$


The loop amplitude corresponding to the diagram shown in Fig. 7(c) is given by ${ }^{6}$

$$
\Pi_{\mu \nu}^{7 c}=\frac{g^{2} N_{c}}{2} \underbrace{}_{\vec{k}} \frac{2(d-2)\left(k^{2}-p \cdot k\right) \delta_{\mu \nu}+\left(2 d^{2}-3 d+4\right)\left[2 k_{\mu} k_{\nu}-\left(p_{\mu} k_{\nu}+k_{\mu} p_{\nu}\right)\right]}{\left(k^{2}-m^{2}\right)\left\{(p-k)^{2}-m^{2}\right\}},
$$

which provides $a_{1}=2(d-2), a_{3}=0, a_{5}=0$, and $n=2$. The loop amplitude from the loop diagram, shown in Fig. 7(d), is

$$
\begin{aligned}
\Pi_{i j}^{7 d} & =\frac{1}{2} g^{2} N_{c} \delta_{i j}\left(d^{2}-3 d+2\right) \sum_{\vec{k}} \frac{1}{\left(k^{2}-m^{2}\right)} \\
& \approx \frac{1}{2} g^{2}\left(d^{2}-3 d+2\right) N_{c} \delta_{i j} \int_{\vec{k}} \frac{1}{2 K}\left(1+n_{B}(K)\right) .
\end{aligned}
$$

Now, there is only one relevant loop diagram involving $A$ and $B$ fields shown in Fig. 8. The amplitude corresponding to this diagram is obtained by neglecting the term $\sim m^{2} / k^{4}$ from the propagators of the field and is obtained as

$$
\begin{gathered}
\Pi_{\mu \nu}^{8}=g^{2} m^{2} N_{c} \bigcup_{\vec{k}} \frac{(2 d-2) \delta_{\mu \nu}}{\left(k^{2}-m^{2}\right)\left[(p-k)^{2}-m^{2}\right]}, \\
\Pi_{i j}^{8}=2 g^{2} m^{2} N_{c}(d-1) \sum_{\vec{k}} \frac{\delta_{i j}}{\left(k^{2}-m^{2}\right)\left[(p-k)^{2}-m^{2}\right]},
\end{gathered}
$$

which gives $a_{3}=2, a_{1}=a_{5}=0$, and $n=1$. Next we consider the ghost sector, which also contributes in the construction of the HTL effective Lagrangian. The loop diagrams corresponding to fields $\omega, \bar{\omega}, \omega^{\mu}, \bar{\omega}^{\mu}$, and $\beta, \bar{\beta}$ are shown in Figs. 9(a)-9(c), respectively.

The loops are formed by the FP ghost of the YM field in Fig. 9(a), vector ghost in Fig. 9(b), and ghost of the vector ghost in Fig. 9(c). The loop amplitude from Fig. 9(a) is found as

$$
\begin{aligned}
\Pi_{\mu \nu}^{9 a} & =g^{2} N_{c} \underbrace{}_{\vec{k}} \frac{(k-p)_{\mu} k_{\nu}}{k^{2}(p-k)^{2}} \\
& =\frac{g^{2} N_{c}}{2} \underbrace{}_{\vec{k}} \frac{2 k_{\mu} k_{\nu}-p_{\mu} p_{\nu}}{k^{2}(p-k)^{2}},
\end{aligned}
$$

where we have used the trick [cf. Eq. (35)] in the last step because the loop integration contains the product of two identical propagators. Comparing with Eq. (44), we see that $a_{1}=a_{3}=0, a_{5}=1$, and $n=1$. Loop amplitude from Fig. $9(\mathrm{~b})$ is

\footnotetext{
${ }^{6}$ See the calculation of the numerator of the integrand in the Appendix B.
}

$$
\begin{aligned}
& \Pi_{\mu \nu}^{9 b}=-g^{2} N_{c} \bigvee_{\vec{k}} \frac{(d-2) p_{\mu} k_{\nu}-k_{\mu} k_{\nu}(d-1)+p_{\nu} k_{\mu}}{k^{2}(p-k)^{2}} \\
& =-\frac{g^{2} N_{c}}{2} \underbrace{}_{\vec{k}} \frac{(d-1) p_{\mu} p_{\nu}-2 k_{\mu} k_{\nu}(d-1)}{k^{2}(p-k)^{2}}, \\
& \Rightarrow \Pi_{i j}^{9 b} \rightarrow g^{2} N_{c}(d-1) \bigvee_{\vec{k}} \frac{k_{i} k_{j}}{k^{2}(p-k)^{2}} .
\end{aligned}
$$

In the last step of the above integration, we have again used the same trick shown in Eq. (35). In comparison with Eq. (44), we see that loop integration contributes to the HTL effective Lagrangian with $a_{1}=a_{3}=0$ and $a_{5}=(d-1)$. The contribution from Fig. 9(a) is the same as that of Fig. 9(c), because of the similarity in the vertices of the trilinear couplings $A \bar{\omega} \omega$ and $A \bar{\beta} \beta$. Hence, adding up the contribution from the ghost sectors, we get

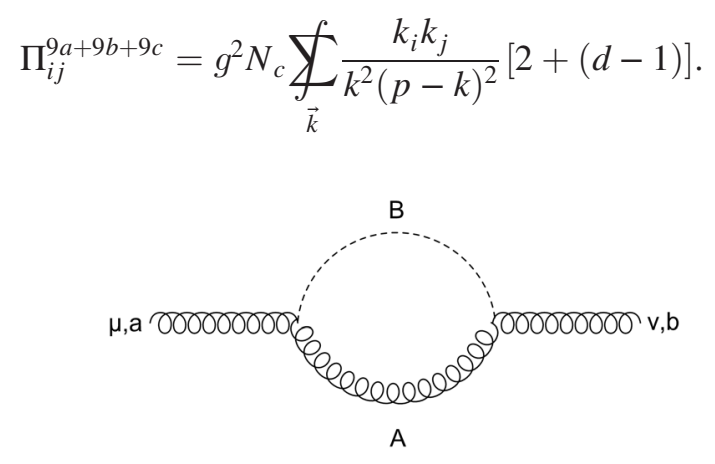

FIG. 8. Loop diagram contains the $A A B$ coupling.

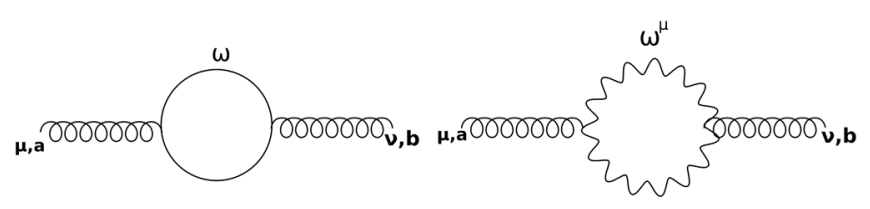

(a)

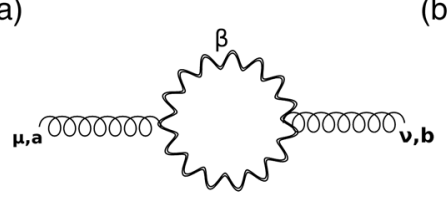

(c)

FIG. 9. Loops formed by (a) FP ghost of YM field, (b) vector ghost, and (c) ghost of vector ghost. 
Comparing the generic expression in Eq. (44) with the above equation, we get only $a_{5}=4$ when $d=3$ and $n=1$. Hence, we obtain the effective action from HTL approximation for topologically massive bosons in $d=3$ dimensions as

$$
\begin{aligned}
\mathcal{L}_{\text {eff }}= & -\frac{1}{4} F_{a}^{\mu \nu} F_{\mu \nu}^{a} \\
& +m^{2} \bigvee_{\vec{k}}\left(A^{\lambda}(k) A_{\lambda}(-k)-\frac{(k \cdot A(-k))(k \cdot A(k))}{k^{2}}\right) \\
& +\int_{K} \tilde{m}^{2}(K) A^{\mu}(K) A_{\mu}(-K) \\
& +m_{E}^{2} \int d \Omega_{v}\left(\frac{1}{\mathcal{V} \cdot D} \mathcal{V}^{\alpha} F_{\alpha \mu}^{a}\right)\left(\frac{1}{\mathcal{V} \cdot D} \mathcal{V}^{\beta} F_{a \beta}^{\mu}\right),
\end{aligned}
$$

where

$$
\begin{gathered}
\tilde{m}^{2}=g^{2} N_{c}\left(\frac{1}{2} \frac{T^{2}}{12}+2 m^{2} I\right), \\
m_{E}^{2} \approx g^{2} N_{c} \frac{T^{2}}{12} .
\end{gathered}
$$

In the final form of the effective action in Eq. (68), we have added the contribution obtained by integrating out the $B$ field from the quadratic part of TMM action for the Lagrangian, given in Eq. (1) (see Appendix C). The effective action in Eq. (68) also contains the contributions from Figs. 7(b) and 7(d). These contributions are added to the coefficient $B$ in Eq. (44) to provide the coefficient $\frac{1}{2}$ of $\frac{T^{2}}{12}$ in Eq. (69).

\section{DISCUSSION}

We have constructed the HTL effective action for the topologically massive gauge theory. In the final form, we have clearly shown how the Debye mass is modified due to the presence of the bare mass of massive gauge bosons. The bare mass puts an infrared cutoff in QCD at finite temperature. The infrared cutoff plays a crucial role in the perturbative analysis of transport coefficients, which are related to the response functions. These were believed to be in the nonperturbation regime in QCD at finite temperature. We have not considered any fermionic interaction with the massive YM gauge bosons. The fermions will have the same trilinear coupling with massive YM fields as they have in massless YM theory. As a consequence, they provide the same contribution in the HTL approximated Lagrangian. There is no conserved local current constructed from a trilinear coupling among fermions and $B_{\mu \nu}$ field. We have not calculated the transport coefficients from the HTL action for topologically massive gauge bosons when they are coupled with fermions. It will be very interesting to find the response functions from a matter coupled TMM at finite temperature.

We also see the other prospects of the TMM at finite temperature. In the massless YM theory at finite temperature, the phase transition can be explained by associating with spontaneously broken symmetry. Massless YM field theory is invariant under the $S U(N) / Z(N)$ group, where $Z(N)$ is the center of the $S U(N)$ group. This symmetry is believed to be spontaneously broken at phase transition, which is described by the vacuum expectation value of the Polyakov loop $\tilde{L}=\frac{1}{N} \operatorname{tr} \mathcal{P}\left(\exp i \oint_{C} A_{0}(\vec{x}, t)\right)$, where $\mathcal{P}$ represents path ordering of the exponent and trace is taken to make $L$ invariant under $S U(N)$ symmetry. Taking the quarks to be static, it can be shown that the implication of phase transition implies the spontaneous breaking of $S U(N)$ symmetry. However, in TMM, there are massive gauge fields, which are in the adjoint representation of the $S U(N)$ group. As a consequence, in the model, we have a more general Polyakov loop

$\tilde{L}^{\text {gen }} \sim \operatorname{tr} \mathcal{P}\left(\exp \left(i \oint A_{0}(\vec{x}, t) d x^{0} \oint_{S} B_{0 i} d x^{0} d x^{i}\right)\right)$,

where the closed path $C$ is the loop and surface $S$ is taken in space-time. The physical significance and the behavior of $\tilde{L}^{\text {gen }}$ near the critical temperature can be investigated thoroughly. It will be also interesting to consider thermal Bethe Salpeter equations from TMM. This may give the dynamics of the bound state massive gauge bosons at finite temperature.

\section{ACKNOWLEDGMENTS}

D. M. thanks the Department of Atomic Energy, Government of India for financial support and R. K. would like to thank UGC, Government of India, New Delhi, for financial support under the PDFSS scheme. We would like to thank Professor S. Mrówczyński for bringing his recent work on the general form of HTL effective action for massless gauge bosons [27] to our notice.

\section{APPENDIX A: FINITENESS OF I APPEARING IN Eq. (43)}

An expression for $I$ in HTL approximation has been derived in the Appendix. We consider the second and third terms appearing in Eq. (32) from which $I$ originates. Omitting some numerical factors, which hardly matter in the computation, we consider the following integration of the second term of Eq. (32) as:

$$
\mathcal{I}_{1}=\int \frac{d^{D} p}{(2 \pi)^{4}} \frac{1}{E_{1} E_{2}} \frac{\left(n_{B}\left(E_{1}\right)-n_{B}\left(E_{2}\right)\right)}{i k_{0 n}+E_{2}-E_{1}} .
$$

Putting $E_{1}=\sqrt{\mathbf{p}^{2}+m^{2}}, E_{2}=\sqrt{(\mathbf{p}-\mathbf{k})^{2}+m^{2}}, n_{B}(E)=$ $\sum_{s=1}^{\infty} e^{-s \beta E}$, and considering the approximations, taken in Eqs. (29) and (30), we get 


$$
\mathcal{I}_{1}=\int \frac{d^{3} p}{(2 \pi)^{3}} \frac{\sum_{s=1}^{\infty}\left(e^{-\beta s \sqrt{\mathbf{p}^{2}+m^{2}}}-e^{-\beta s \sqrt{(\mathbf{p}-\mathbf{k})^{2}+m^{2}}}\right)}{\left(P+\frac{m^{2}}{2 P}+\mathcal{O}\left(\frac{m^{4}}{P^{2}}\right)\right)\left(P-\mathbf{k} \cdot \mathbf{v}+\frac{m^{2}}{2 P}+\mathcal{O}\left(\frac{\left(m^{2}-\mathbf{k} \cdot \mathbf{v}\right)^{2}}{P^{2}}\right)\right)} \frac{1}{i k_{0 n}-\mathbf{k} \cdot \mathbf{v}} .
$$

Neglecting $K$ and $m$ with respect to $P$, the integral $\mathcal{I}_{1}$ reduces (in $D=3$ ) to the following form:

$$
\mathcal{I}_{1} \approx \int \frac{d \Omega}{(2 \pi)^{3}} \frac{1}{i k_{0 n}-\mathbf{k} \cdot \mathbf{v}} \sum_{s=1}^{\infty}\left[\int_{0}^{\infty}\left(e^{-\beta s \sqrt{\mathbf{p}^{2}+m^{2}}}-e^{-\beta s \sqrt{(\mathbf{p}-\mathbf{k})^{2}+m^{2}}}\right) d P+\mathcal{T}_{1}\left(m^{2}, K, s\right)\right],
$$

where $\mathcal{T}_{1}\left(m^{2}, K, s\right)$ represent the finite terms that depend on $m^{2}, K$, and $s$. Using the result

$$
\int_{0}^{\infty} x^{\nu-1} e^{-\frac{\alpha}{x}-\gamma x} d x=2\left(\frac{\alpha}{\gamma}\right)^{\nu / 2} K_{\nu}(2 \sqrt{\alpha \gamma}), \quad[\operatorname{Re} \alpha>0, \operatorname{Re} \gamma>0]
$$

$\mathcal{I}_{1}$ can be approximated as

$$
\mathcal{I}_{1} \approx \sum_{s=1}^{\infty}\left[\int \frac{d \Omega}{(2 \pi)^{3}} \frac{m \sqrt{2} K_{1}(\sqrt{2} s m \beta)}{i k_{0 n}-\mathbf{k} \cdot \mathbf{v}}\left(1-e^{s \beta \mathbf{k} \cdot \mathbf{v}}\right)+\mathcal{T}_{1}\left(m^{2}, K, s\right)\right] .
$$

Similarly, from the third term of Eq. (32), the contribution becomes

$$
\mathcal{I}_{2} \approx-\sum_{s=1}^{\infty}\left[\int \frac{d \Omega}{(2 \pi)^{3}} \frac{m \sqrt{2} K_{1}(\sqrt{2} s m \beta)}{i k_{0 n}+\mathbf{k} \cdot \mathbf{v}}\left(1-e^{s \beta \mathbf{k} \cdot \mathbf{v}}\right)+\mathcal{T}_{2}\left(m^{2}, K, s\right)\right] .
$$

Hence, we have

$$
I=\mathcal{I}_{1}+\mathcal{I}_{2} \approx\left[\sum_{s=1}^{\infty} \int \frac{d \Omega}{(2 \pi)^{3}} \frac{2 \sqrt{2} \mathbf{k} \cdot \mathbf{v}}{k_{0 n}^{2}+(\mathbf{k} \cdot \mathbf{v})^{2}} m K_{1}(\sqrt{2} s m \beta)\left(e^{s \beta \mathbf{k} \cdot \mathbf{v}}-\right)+\mathcal{T}\left(m^{2}, K, s\right)\right] .
$$

It is clear that this is a convergent sum because of the behavior of $K_{1}(\sqrt{2} s \beta m)$ in the limit $s \rightarrow \infty$ and $m \neq 0, s \neq 0$. In summary, our purpose was to see how the integration over $p$ is convergent and this has been shown in a clear way here. After the integration over a solid angle $\Omega$, we get

$$
I \approx 2 \sqrt{2} \frac{1}{(2 \pi)^{2} K} \sum_{s=1}^{\infty} m K_{1}(\sqrt{2} s m \beta) \operatorname{Re}\left[e^{i s \beta k_{0 n}} E_{1}\left(s \beta\left\{K+i k_{0 n}\right\}\right)-E_{1}\left(s \beta\left\{-K+i k_{0 n}\right\}\right)\right],
$$

where $E_{1}(x)$ is an incomplete Gamma function [63]. In reaching the above result, we have used [63]

$$
\int \frac{x e^{x}}{x^{2}+a^{2}}=-\operatorname{Re}\left(e^{i a} E_{1}(-x+i a)+\text { const }\right), \quad[a>0],
$$

where $E_{1}(x)=\int_{x}^{\infty} \frac{e^{-t}}{t} d t$.

\section{APPENDIX B: CALCULATION OF AMPLITUDE OF FIG. 7(c)}

The amplitude of Fig. 10 is given by

$$
\begin{aligned}
\mathcal{M}= & \frac{1}{16}\left[(2 k-p)_{\mu} \eta_{\rho[\alpha} \eta_{\beta] \sigma}+k_{[\rho} \eta_{\sigma][\alpha} \eta_{\beta] \mu}-\tilde{k}_{[\alpha} \eta_{\beta][\rho} \eta_{\sigma] \mu}\right]\left(\frac{\eta^{\alpha\left[\alpha^{\prime}\right.} \eta^{\left.\beta^{\prime}\right] \beta}}{k^{2}-m^{2}}+\frac{m^{2}}{k^{4}} \frac{k^{[\alpha} k^{\left[\beta^{\prime}\right.} \eta^{\left.\left.\alpha^{\prime}\right] \beta\right]}}{k^{2}-m^{2}}\right) \\
& \times\left(\frac{\eta^{\rho\left[\rho^{\prime}\right.} \eta^{\left.\sigma^{\prime}\right] \sigma}}{\tilde{k}^{2}-m^{2}}+\frac{m^{2}}{\tilde{k}^{4}} \frac{\tilde{k}^{[\rho} k^{\left[\sigma^{\prime}\right.} \eta^{\left.\left.\rho^{\prime}\right] \sigma\right]}}{\tilde{k}^{2}-m^{2}}\right)\left[(2 k-p)_{\nu} \eta_{\alpha^{\prime}\left[\rho^{\prime}\right.} \eta_{\left.\sigma^{\prime}\right] \beta^{\prime}}+k_{\left[\rho^{\prime}\right.} \eta_{\left.\sigma^{\prime}\right]\left[\alpha^{\prime}\right.} \eta_{\left.\beta^{\prime}\right] \nu}-\tilde{k}_{\left[\alpha^{\prime}\right.} \eta_{\left.\beta^{\prime}\right]\left[\rho^{\prime}\right.} \eta_{\left.\sigma^{\prime}\right] \nu}\right],
\end{aligned}
$$


where $\tilde{k}$ is given by

$$
\tilde{k}=p-k
$$

Now we will use the following property in the amplitude:

$$
A^{[\mu \nu]} \eta_{\alpha[\mu} \eta_{\nu] \beta}=2 A^{\mu \nu} \eta_{\alpha[\mu} \eta_{\nu] \beta},
$$

so that we get

$$
\begin{aligned}
& \mathcal{M}=\frac{1}{16}\left[2(2 k-p)_{\mu} \eta_{\rho \alpha} \eta_{\beta \sigma}+2 k_{[\rho} \eta_{\sigma] \alpha} \eta_{\beta \mu}-2 \tilde{k}_{\alpha} \eta_{\beta[\rho} \eta_{\sigma] \mu}\right]\left(\frac{\eta^{\alpha\left[\alpha^{\prime}\right.} \eta^{\left.\beta^{\prime}\right] \beta}}{k^{2}-m^{2}}+\frac{m^{2}}{k^{4}} \frac{k^{[\alpha} k^{\left[\beta^{\prime}\right.} \eta^{\left.\left.\alpha^{\prime}\right] \beta\right]}}{k^{2}-m^{2}}\right) \\
& \times\left(\frac{\eta^{\rho\left[\rho^{\prime}\right.} \eta^{\left.\sigma^{\prime}\right] \sigma}}{\tilde{k}^{2}-m^{2}}+\frac{m^{2}}{\tilde{k}^{4}} \frac{\tilde{k}^{[\rho} k^{\left[\sigma^{\prime}\right.} \eta^{\left.\left.\rho^{\prime}\right] \sigma\right]}}{\tilde{k}^{2}-m^{2}}\right)\left[2(2 k-p)_{\nu} \eta_{\alpha^{\prime} \rho^{\prime}} \eta_{\sigma^{\prime} \beta^{\prime}}+2 k_{\rho^{\prime}} \eta_{\sigma^{\prime}\left[\alpha^{\prime}\right.} \eta_{\left.\beta^{\prime}\right] \nu}-2 \tilde{k}_{\left[\alpha^{\prime}\right.} \eta_{\left.\beta^{\prime}\right] \rho^{\prime}} \eta_{\sigma^{\prime} \nu}\right] \\
& =\frac{4}{16}\left[(2 k-p)_{\mu} \eta_{\rho \alpha} \eta_{\beta \sigma}+k_{[\rho} \eta_{\sigma] \alpha} \eta_{\beta \mu}-\tilde{k}_{\alpha} \eta_{\beta[\rho} \eta_{\sigma] \mu}\right]\left(\frac{\eta^{\alpha\left[\alpha^{\prime}\right.} \eta^{\left.\beta^{\prime}\right] \beta}}{k^{2}-m^{2}}+\frac{m^{2}}{k^{4}} \frac{k^{[\alpha} k^{\left[\beta^{\prime}\right.} \eta^{\left.\left.\alpha^{\prime}\right] \beta\right]}}{k^{2}-m^{2}}\right) \\
& \times\left(\frac{\eta^{\rho\left[\rho^{\prime}\right.} \eta^{\left.\sigma^{\prime}\right] \sigma}}{\tilde{k}^{2}-m^{2}}+\frac{m^{2}}{\tilde{k}^{4}} \frac{\tilde{k}^{[\rho} k^{\left[\sigma^{\prime}\right.} \eta^{\left.\left.\rho^{\prime}\right] \sigma\right]}}{\tilde{k}^{2}-m^{2}}\right)\left[(2 k-p)_{\nu} \eta_{\alpha^{\prime} \rho^{\prime}} \eta_{\sigma^{\prime} \beta^{\prime}}+k_{\rho^{\prime}} \eta_{\sigma^{\prime}\left[\alpha^{\prime}\right.} \eta_{\left.\beta^{\prime}\right] \nu}-\tilde{k}_{\left[\alpha^{\prime}\right.} \eta_{\left.\beta^{\prime}\right] \rho^{\prime}} \eta_{\sigma^{\prime} \nu}\right] \\
& =\frac{4}{16}\left[(2 k-p)_{\mu} \eta_{\rho \alpha} \eta_{\beta \sigma}+2 k_{\rho} \eta_{\sigma \alpha} \eta_{\beta \mu}-2 \tilde{k}_{\alpha} \eta_{\beta \rho} \eta_{\sigma \mu}\right]\left(\frac{\eta^{\alpha\left[\alpha^{\prime}\right.} \eta^{\left.\beta^{\prime}\right] \beta}}{k^{2}-m^{2}}+\frac{m^{2}}{k^{4}} \frac{k^{[\alpha} k^{\left[\beta^{\prime}\right.} \eta^{\left.\left.\alpha^{\prime}\right] \beta\right]}}{k^{2}-m^{2}}\right) \\
& \times\left(\frac{\eta^{\rho\left[\rho^{\prime}\right.} \eta^{\left.\sigma^{\prime}\right] \sigma}}{\tilde{k}^{2}-m^{2}}+\frac{m^{2}}{\tilde{k}^{4}} \frac{\tilde{k}^{[\rho} k^{\left[\sigma^{\prime}\right.} \eta^{\left.\left.\rho^{\prime}\right] \sigma\right]}}{\tilde{k}^{2}-m^{2}}\right)\left[(2 k-p)_{\nu} \eta_{\alpha^{\prime} \rho^{\prime}} \eta_{\sigma^{\prime} \beta^{\prime}}+2 k_{\rho^{\prime}} \eta_{\sigma^{\prime} \alpha^{\prime}} \eta_{\beta^{\prime} \nu}-2 \tilde{k}_{\alpha^{\prime}} \eta_{\beta^{\prime} \rho^{\prime}} \eta_{\sigma^{\prime} \nu}\right] .
\end{aligned}
$$

Now we ignore $O\left(\mathrm{~m}^{2} / \mathrm{k}^{4}\right)$ and $O\left(\mathrm{~m}^{2} / \mathrm{k}^{6}\right)$ terms to get

$$
\begin{aligned}
\mathcal{M}= & \frac{4}{16\left(k^{2}-m^{2}\right)\left(\tilde{k}^{2}-m^{2}\right)}\left[(2 k-p)_{\mu} \eta_{\rho \alpha} \eta_{\beta \sigma}+2 k_{\rho} \eta_{\sigma \alpha} \eta_{\beta \mu}-2 \tilde{k}_{\alpha} \eta_{\beta \rho} \eta_{\sigma \mu}\right]\left(\eta^{\alpha\left[\alpha^{\prime}\right.} \eta^{\left.\beta^{\prime}\right] \beta}\right) \\
& \times\left(\eta^{\rho\left[\rho^{\prime}\right.} \eta^{\left.\sigma^{\prime}\right] \sigma}\right)\left[(2 k-p)_{\nu} \eta_{\alpha^{\prime} \rho^{\prime}} \eta_{\sigma^{\prime} \beta^{\prime}}+2 k_{\rho^{\prime}} \eta_{\sigma^{\prime} \alpha^{\prime}} \eta_{\beta^{\prime} \nu}-2 \tilde{k}_{\alpha^{\prime}} \eta_{\left.\beta^{\prime} \rho^{\prime} \eta_{\sigma^{\prime} \nu}\right]}\right. \\
= & \frac{4}{16\left(k^{2}-m^{2}\right)\left(\tilde{k}^{2}-m^{2}\right)}\left[(2 k-p)_{\mu} \delta_{\rho}^{\left[\alpha^{\prime}\right.} \delta_{\sigma}^{\left.\beta^{\prime}\right]}+2 k_{\rho} \delta_{\sigma}^{\left[\alpha^{\prime}\right.} \delta_{\mu}^{\left.\beta^{\prime}\right]}-2 \tilde{k}^{\left[\alpha^{\prime}\right.} \delta_{\rho}^{\left.\beta^{\prime}\right]} \eta_{\sigma \mu}\right] \\
& \times\left[(2 k-p)_{\nu} \delta_{\alpha^{\prime}}^{[\rho} \delta_{\beta^{\prime}}^{\sigma]}+2 k^{[\rho} \delta_{\alpha^{\prime}}^{\sigma]} \eta_{\beta^{\prime} \nu}-2 \tilde{k}_{\alpha^{\prime}} \delta_{\beta^{\prime}}^{[\rho} \delta_{\nu}^{\sigma]}\right]
\end{aligned}
$$

Let us denote the first and second square brackets by I and II, respectively, i.e.,

$$
\begin{gathered}
\mathrm{I}=\left[(2 k-p)_{\mu} \delta_{\rho}^{\left[\alpha^{\prime}\right.} \delta_{\sigma}^{\left.\beta^{\prime}\right]}+2 k_{\rho} \delta_{\sigma}^{\left[\alpha^{\prime}\right.} \delta_{\mu}^{\left.\beta^{\prime}\right]}-2 \tilde{k}^{\left[\alpha^{\prime}\right.} \delta_{\rho}^{\left.\beta^{\beta^{\prime}}\right]} \eta_{\sigma \mu}\right], \\
\mathrm{II}=\left[(2 k-p)_{\nu} \delta_{\alpha^{\prime}}^{[\rho} \delta_{\beta^{\prime}}^{\sigma]}+2 k^{[\rho} \delta_{\alpha^{\prime}}^{\sigma]} \eta_{\beta^{\prime} \nu}-2 \tilde{k}_{\alpha^{\prime}} \delta_{\beta^{\prime}}^{[\rho} \delta_{\nu}^{\sigma]}\right] .
\end{gathered}
$$

The first term in I and first term in II are antisymmetric with respect to $\alpha^{\prime}$ and $\beta^{\prime}$, so that we have

$$
\mathrm{I} \times \mathrm{II}=\left[2(2 k-p)_{\mu} \delta_{\rho}^{\alpha^{\prime}} \delta_{\sigma}^{\beta^{\prime}}+2 k_{\rho} \delta_{\sigma}^{\left[\alpha^{\prime}\right.} \delta_{\mu}^{\left.\beta^{\prime}\right]}-2 \tilde{k}^{\left[\alpha^{\prime}\right.} \delta_{\rho}^{\left.\beta^{\prime}\right]} \eta_{\sigma \mu}\right]\left[(2 k-p)_{\nu} \delta_{\alpha^{\prime}}^{[\rho} \delta_{\beta^{\prime}}^{\sigma]}+2 k^{[\rho} \delta_{\alpha^{\prime}}^{\sigma]} \eta_{\beta^{\prime} \nu}-2 \tilde{k}_{\alpha^{\prime}} \delta_{\beta^{\prime}}^{[\rho} \delta_{\nu}^{\sigma]}\right] .
$$

Again, the first term in first square bracket and first term in the second square bracket above are antisymmetric with respect to $\rho$ and $\sigma$, so that we have

$$
\mathrm{I} \times \mathrm{II}=\left[2(2 k-p)_{\mu} \delta_{\rho}^{\alpha^{\prime}} \delta_{\sigma}^{\beta^{\prime}}+2 k_{\rho} \delta_{\sigma}^{\left[\alpha^{\prime}\right.} \delta_{\mu}^{\left.\beta^{\prime}\right]}-2 \tilde{k}^{\left[\alpha^{\prime}\right.} \delta_{\rho}^{\left.\beta^{\prime}\right]} \eta_{\sigma \mu}\right]\left[2(2 k-p)_{\nu} \delta_{\alpha^{\prime}}^{\rho} \delta_{\beta^{\prime}}^{\sigma}+2 k^{[\rho} \delta_{\alpha^{\prime}}^{\sigma]} \eta_{\beta^{\prime} \nu}-2 \tilde{k}_{\alpha^{\prime}} \delta_{\beta^{\prime}}^{[\rho} \delta_{\nu}^{\sigma]}\right],
$$

which implies

$$
\mathrm{I} \times \mathrm{II}=4\left[(2 k-p)_{\mu} \delta_{\rho}^{\alpha^{\prime}} \delta_{\sigma}^{\beta^{\prime}}+k_{\rho} \delta_{\sigma}^{\left[\alpha^{\prime}\right.} \delta_{\mu}^{\left.\beta^{\prime}\right]}-\tilde{k}^{\left[\alpha^{\prime}\right.} \delta_{\rho}^{\left.\beta^{\prime}\right]} \eta_{\sigma \mu}\right]\left[(2 k-p)_{\nu} \delta_{\alpha^{\prime}}^{\rho} \delta_{\beta^{\prime}}^{\sigma}+k^{[\rho} \delta_{\alpha^{\prime}}^{\sigma]} \eta_{\beta^{\prime} \nu}-\tilde{k}_{\alpha^{\prime}} \delta_{\beta^{\prime}}^{[\rho} \delta_{\nu}^{\sigma]}\right]
$$


Hence, the amplitude becomes

$$
\mathcal{M}=\frac{1}{\left(k^{2}-m^{2}\right)\left(\tilde{k}^{2}-m^{2}\right)}\left[(2 k-p)_{\mu} \delta_{\rho}^{\alpha^{\prime}} \delta_{\sigma}^{\beta^{\prime}}+k_{\rho} \delta_{\sigma}^{\left[\alpha^{\prime}\right.} \delta_{\mu}^{\left.\beta^{\prime}\right]}-\tilde{k}^{\left[\alpha^{\prime}\right.} \delta_{\rho}^{\left.\beta^{\prime}\right]} \eta_{\sigma \mu}\right]\left[(2 k-p)_{\nu} \delta_{\alpha^{\prime}}^{\rho} \delta_{\beta^{\prime}}^{\sigma}+k^{[\rho} \delta_{\alpha^{\prime}}^{\sigma]} \eta_{\beta^{\prime} \nu}-\tilde{k}_{\alpha^{\prime}} \delta_{\beta^{\prime}}^{[\rho} \delta_{\nu}^{\sigma]}\right] .
$$

Again, let

$$
\begin{aligned}
& \mathrm{I}=\left[(2 k-p)_{\mu} \delta_{\rho}^{\alpha^{\prime}} \delta_{\sigma}^{\beta^{\prime}}+k_{\rho} \delta_{\sigma}^{\left[\alpha^{\prime}\right.} \delta_{\mu}^{\left.\beta^{\prime}\right]}-\tilde{k}^{\left[\alpha^{\prime}\right.} \delta_{\rho}^{\left.\beta^{\prime}\right]} \eta_{\sigma \mu}\right], \\
& \mathrm{II}=\left[(2 k-p)_{\nu} \delta_{\alpha^{\prime}}^{\rho} \delta_{\beta^{\prime}}^{\sigma}+k^{[\rho} \delta_{\alpha^{\prime}}^{\sigma]} \eta_{\beta^{\prime} \nu}-\tilde{k}_{\alpha^{\prime}} \delta_{\beta^{\prime}}^{[\rho} \delta_{\nu}^{\sigma]}\right] .
\end{aligned}
$$

Also let $\mathrm{I}_{i}$ denote the $i$ th term in $\mathrm{I}$, so that we have

$$
\begin{gathered}
\mathrm{I}=\mathrm{I}_{1}+\mathrm{I}_{2}-\mathrm{I}_{3}, \\
\mathrm{II}=\mathrm{II}_{1}+\mathrm{II}_{2}-\mathrm{II}_{3},
\end{gathered}
$$

and

$$
\begin{aligned}
\mathrm{I}_{1} \times \mathrm{II}_{1} & =(2 k-p)_{\mu} \delta_{\rho}^{\alpha^{\prime}} \delta_{\sigma}^{\beta^{\prime}} \times(2 k-p)_{\nu} \delta_{\alpha^{\prime}}^{\rho} \delta_{\beta^{\prime}}^{\sigma} \\
& =d^{2}(2 k-p)_{\mu}(2 k-p)_{\nu} \\
& \approx d^{2}\left[4 k_{\mu} k_{\nu}-2\left(k_{\mu} p_{\nu}+k_{\nu} p_{\mu}\right)\right]
\end{aligned}
$$

$$
\begin{aligned}
\mathrm{I}_{1} \times \mathrm{II}_{2} & =(2 k-p)_{\mu} \delta_{\rho}^{\alpha^{\prime}} \delta_{\sigma}^{\beta^{\prime}} \times k^{[\rho} \delta_{\alpha^{\prime}}^{\sigma]} \eta_{\beta^{\prime} \nu} \\
& =(2 k-p)_{\mu} \times k^{\left[\alpha^{\prime}\right.} \delta_{\alpha^{\prime}}^{\sigma]} \eta_{\sigma \nu} \\
& =(2 k-p)_{\mu} \times\left(k^{\alpha^{\prime}} \delta_{\alpha^{\prime}}^{\sigma}-k^{\sigma} \delta_{\alpha^{\prime}}^{\alpha^{\prime}}\right) \eta_{\sigma \nu} \\
& =(1-d)\left(2 k_{\mu} k_{\nu}-p_{\mu} k_{\nu}\right),
\end{aligned}
$$

$$
\begin{aligned}
\mathrm{I}_{1} \times \mathrm{II}_{3} & =(2 k-p)_{\mu} \delta_{\rho}^{\alpha^{\prime}} \delta_{\sigma}^{\beta^{\prime}} \times \tilde{k}_{\alpha^{\prime}} \delta_{\beta^{\prime}}^{[\rho} \sigma_{\nu}^{\sigma]} \\
& =(2 k-p)_{\mu} \tilde{k}_{\rho} \delta_{\sigma}^{\rho} \delta_{\nu}^{\sigma]} \\
& =(2 k-p)_{\mu} \tilde{k}_{\rho}\left(\delta_{\sigma}^{\rho} \delta_{\nu}^{\sigma}-\delta_{\sigma}^{\sigma} \delta_{\nu}^{\rho}\right) \\
& =(1-d)\left(2 k_{\mu} \tilde{k}_{\nu}-p_{\mu} \tilde{k}_{\nu}\right) \\
& \approx(1-d)\left(2 k_{\mu} p_{\nu}-2 k_{\mu} k_{\nu}+p_{\mu} k_{\nu}\right),
\end{aligned}
$$

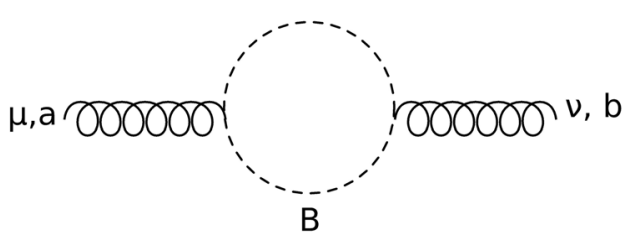

FIG. 10. Loop diagram contains the $A B B$ coupling.

$$
\begin{aligned}
\mathrm{I}_{2} \times \mathrm{II}_{1} & =k_{\rho} \delta_{\sigma}^{\left[\alpha^{\prime}\right.} \delta_{\mu}^{\left.\beta^{\prime}\right]} \times(2 k-p)_{\nu} \delta_{\alpha^{\prime}}^{\rho} \delta_{\beta^{\prime}}^{\sigma} \\
& =k_{\alpha^{\prime}} \delta_{\beta^{\prime}}^{\left[\alpha^{\prime}\right.} \delta_{\mu}^{\left.\beta^{\prime}\right]} \times(2 k-p)_{\nu} \\
& =k_{\alpha^{\prime}}(2 k-p)_{\nu}\left(\delta_{\beta^{\prime}}^{\alpha^{\prime}} \delta_{\mu}^{\beta^{\prime}}-\delta_{\beta^{\prime}}^{\beta^{\prime}} \delta_{\mu}^{\alpha^{\prime}}\right) \\
& =(1-d) k_{\mu}(2 k-p)_{\nu} \\
& =(1-d)\left[2 k_{\mu} k_{\nu}-k_{\mu} p_{\nu}\right],
\end{aligned}
$$

$\mathrm{I}_{2} \times \mathrm{II}_{2}=k_{\rho} \delta_{\sigma}^{\left[\alpha^{\prime}\right.} \delta_{\mu}^{\left.\beta^{\prime}\right]} \times k^{[\rho} \delta_{\alpha^{\prime}}^{\sigma]} \eta_{\beta^{\prime} \nu}$

$$
\begin{aligned}
& =k_{\rho}\left(\delta_{\sigma}^{\alpha^{\prime}} \delta_{\mu}^{\beta^{\prime}}-\delta_{\sigma}^{\beta^{\prime}} \delta_{\mu}^{\alpha^{\prime}}\right)\left(k^{\rho} \delta_{\alpha^{\prime}}^{\sigma}-k^{\sigma} \delta_{\alpha^{\prime}}^{\rho}\right) \eta_{\beta^{\prime} \nu} \\
& =\left(\delta_{\sigma}^{\alpha^{\prime}} \eta_{\mu \nu}-\eta_{\sigma \nu} \delta_{\mu}^{\alpha^{\prime}}\right)\left(k^{2} \delta_{\alpha^{\prime}}^{\sigma}-k^{\sigma} k_{\alpha^{\prime}}\right) \\
& =(d-2) k^{2} \eta_{\mu \nu}+k_{\mu} k_{\nu},
\end{aligned}
$$

$\mathrm{I}_{2} \times \mathrm{II}_{3}=k_{\rho} \delta_{\sigma}^{\left[\alpha^{\prime}\right.} \delta_{\mu}^{\left.\beta^{\prime}\right]} \times \tilde{k}_{\alpha^{\prime}} \delta_{\beta^{\prime}}^{[\rho} \delta_{\nu}^{\sigma]}$

$$
\begin{aligned}
& =k_{\rho} \tilde{k}_{\alpha^{\prime}}\left(\delta_{\sigma}^{\alpha^{\prime}} \delta_{\mu}^{\beta^{\prime}}-\delta_{\sigma}^{\beta^{\prime}} \delta_{\mu}^{\alpha^{\prime}}\right)\left(\delta_{\beta^{\prime}}^{\rho} \delta_{\nu}^{\sigma}-\delta_{\beta^{\prime}}^{\sigma} \delta_{\nu}^{\rho}\right) \\
& =\left(\tilde{k}_{\sigma} \delta_{\mu}^{\beta^{\prime}}-\tilde{k}_{\mu} \delta_{\sigma}^{\beta^{\prime}}\right)\left(k_{\beta^{\prime}} \delta_{\nu}^{\sigma}-k_{\nu} \delta_{\beta^{\prime}}^{\sigma}\right) \\
& =(d-2) \tilde{k}_{\mu} k_{\nu}+\tilde{k}_{\nu} k_{\mu} \\
& =(d-2) p_{\mu} k_{\nu}+p_{\nu} k_{\mu}-(d-1) k_{\mu} k_{\nu},
\end{aligned}
$$

$$
\begin{aligned}
\mathrm{I}_{3} \times \mathrm{II}_{1} & =\tilde{k}^{\left[\alpha^{\prime}\right.} \delta_{\rho}^{\left.\beta^{\prime}\right]} \eta_{\sigma \mu} \times(2 k-p)_{\nu} \delta_{\alpha^{\prime}}^{\rho} \delta_{\beta^{\prime}}^{\sigma} \\
& =(2 k-p)_{\nu} \tilde{k}^{[\rho} \delta_{\rho}^{\left.\beta^{\prime}\right]} \eta_{\beta^{\prime} \mu} \\
& =(1-d) \tilde{k}_{\mu}(2 k-p)_{\nu} \\
& \approx(1-d)\left[2 p_{\mu} k_{\nu}-2 k_{\mu} k_{\nu}+k_{\mu} p_{\nu}\right],
\end{aligned}
$$

$$
\begin{aligned}
\mathrm{I}_{3} \times \mathrm{II}_{2} & =\tilde{k}^{\left[\alpha^{\prime}\right.} \delta_{\rho}^{\left.\beta^{\prime}\right]} \eta_{\sigma \mu} \times k^{[\rho} \delta_{\alpha^{\prime}}^{\sigma]} \eta_{\beta^{\prime} \nu} \\
& =\left(\tilde{k}^{\alpha^{\prime}} \delta_{\rho}^{\beta^{\prime}}-\tilde{k}^{\beta^{\prime}} \delta_{\rho}^{\alpha^{\prime}}\right)\left(k^{\rho} \delta_{\alpha^{\prime}}^{\sigma}-k^{\sigma} \delta_{\alpha^{\prime}}^{\rho}\right) \eta_{\sigma \mu} \eta_{\beta^{\prime} \nu} \\
& =\left(\tilde{k}^{\alpha^{\prime}} \eta_{\rho \nu}-\tilde{k}_{\nu} \delta_{\rho}^{\alpha^{\prime}}\right)\left(k^{\rho} \eta_{\alpha^{\prime} \mu}-k_{\mu} \delta_{\alpha^{\prime}}^{\rho}\right) \\
& =\tilde{k}_{\mu} k_{\nu}+(d-2) \tilde{k}_{\nu} k_{\mu} \\
& =p_{\mu} k_{\nu}+(d-2) p_{\nu} k_{\mu}+(1-d) k_{\mu} k_{\nu},
\end{aligned}
$$

$$
\begin{aligned}
\mathrm{I}_{3} \times \mathrm{II}_{3}= & \tilde{k}^{\left[\alpha^{\prime}\right.} \delta_{\rho}^{\left.\beta^{\prime}\right]} \eta_{\sigma \mu} \times \tilde{k}_{\alpha^{\prime}} \delta_{\beta^{\prime}}^{[\rho} \delta_{\nu}^{\sigma]} \\
= & \left(\tilde{k}^{\alpha^{\prime}} \delta_{\rho}^{\beta^{\prime}}-\tilde{k}^{\beta^{\prime}} \delta_{\rho}^{\alpha^{\prime}}\right)\left(\delta_{\beta^{\prime}}^{\rho} \delta_{\nu}^{\sigma}-\delta_{\beta^{\prime}}^{\sigma} \delta_{\nu}^{\rho}\right) \eta_{\sigma \mu} \tilde{k}_{\alpha^{\prime}} \\
= & \left(\tilde{k}^{2} \delta_{\rho}^{\beta^{\prime}}-\tilde{k}^{\beta^{\prime}} \tilde{k}_{\rho}\right)\left(\delta_{\beta^{\prime}}^{\rho} \eta_{\mu \nu}-\eta_{\beta^{\prime} \mu} \delta_{\nu}^{\rho}\right) \\
= & (d-2) \tilde{k}^{2} \eta_{\mu \nu}+\tilde{k}_{\mu} \tilde{k}_{\nu} \\
\approx & (d-2)\left(k^{2}-2 p \cdot k\right) \eta_{\mu \nu} \\
& +k_{\mu} k_{\nu}-\left(p_{\mu} k_{\nu}+k_{\mu} p_{\nu}\right) .
\end{aligned}
$$


The amplitude is finally given by

$$
\begin{aligned}
\mathcal{M}= & \mathrm{I}_{1} \times \mathrm{II}_{1}+\mathrm{I}_{1} \times \mathrm{II}_{2}-\mathrm{I}_{1} \times \mathrm{II}_{3}+\mathrm{I}_{2} \times \mathrm{II}_{1} \\
& +\mathrm{I}_{2} \times \mathrm{II}_{2}-\mathrm{I}_{2} \times \mathrm{II}_{3}-\mathrm{I}_{3} \times \mathrm{II}_{1} \\
& -\mathrm{I}_{3} \times \mathrm{II}_{2}+\mathrm{I}_{3} \times \mathrm{II}_{3} \\
= & 2(d-2)\left(k^{2}-p \cdot k\right) \eta_{\mu \nu} \\
& +\left(2 d^{2}-3 d+4\right)\left[2 k_{\mu} k_{\nu}-\left(p_{\mu} k_{\nu}+k_{\mu} p_{\nu}\right)\right] .
\end{aligned}
$$

\section{APPENDIX C: "INTEGRATING OUT" $B$ FIELD AT QUADRATIC LEVEL}

We have already integrated out the hard modes of the $B$ field from the non-Abelian TMM by considering the trilinear and quartic interactions among $B$ and $A$ fields. However, in the final form of the effective action in
Eq. (68), we have to add classical action, where the $B$ field is integrated out from its quadratic part. We consider the quadratic part

$\mathcal{L}=-\frac{1}{4} F^{\mu \nu} F_{\mu \nu}+\frac{1}{12} H^{\mu \nu \lambda} H_{\mu \nu \lambda}+\frac{m}{4} \varepsilon^{\mu \nu \rho \lambda} F_{\mu \nu} B_{\rho \lambda}$,

where we have suppressed the gauge group indices. Introducing the gauge-fixing term

$$
\mathcal{L}_{G F}=\frac{1}{2 \eta}\left(\partial_{\mu} B^{\mu \nu}\right)^{2}
$$

in the Lagrangian in Eq. (C1), where $\eta$ is a gauge-fixing parameter, we can find the two-point function of the $B$ field. Hence, we can write the action corresponding to the above Lagrangian density in Eq. (C1) as

$$
\begin{aligned}
\mathcal{S}= & \int d^{4} x\left(\frac{1}{2} \int d^{4} y A_{\mu}(x)\left(\eta^{\mu \nu} \square-\partial^{\mu} \partial^{\nu}\right) \delta^{4}(x-y) A_{\nu}(y)\right. \\
& \left.-\frac{1}{4} \int d^{4} y B_{\mu \nu}(x) \Delta^{\mu \nu, \rho \lambda}(x, y, \eta) B_{\rho \lambda}(y)+\frac{m}{2} \int d^{4} y j^{\mu \nu}(x) \delta^{4}(x-y) B_{\mu \nu}(y)\right),
\end{aligned}
$$

where $\Delta(x, y, \eta)$ is the inverse of the two-point function of $B$ field at the tree level and it has mass dimension $[\Delta]=6$ due to the inclusion of the Dirac delta function, where $j^{\alpha \beta}=\frac{1}{2} \varepsilon^{\alpha \beta \rho \lambda} F_{\rho \lambda}$. We can reexpress the above expression as

$$
\begin{aligned}
\mathcal{S}= & -\int d^{4} x \frac{1}{4} F^{\mu \nu} F_{\mu \nu}-\frac{1}{4} \int d^{4} x\left[\int d^{4} y\left(B_{\mu \nu}(x)+\frac{m}{2} \int d^{4} z j^{\alpha \beta}(z) \Delta_{\alpha \beta, \mu \nu}^{-1}(z, x, \eta)\right) \Delta^{\mu \nu, \rho \lambda}(x, y, \eta)\right. \\
& \left.\times\left(B_{\rho \lambda}(y)+\frac{m}{2} \int d^{4} z \Delta_{\rho \lambda, \alpha \beta}^{-1}(y, z, \eta) j^{\alpha \beta}(z)\right)\right]+\frac{m^{2}}{4} \int d^{4} k j^{\alpha \beta}(-k) \Delta_{\alpha \beta, \alpha^{\prime} \beta^{\prime}}^{-1}(k) j^{\alpha^{\prime} \beta^{\prime}}(k) .
\end{aligned}
$$

The last term appears from the following steps:

$$
\begin{aligned}
& \frac{m^{2}}{16} \int d^{4} x d^{4} y d^{4} z d^{4} z^{\prime} j^{\alpha \beta}(z) \Delta_{\alpha \beta, \mu \nu}^{-1}(z, x, \eta) \Delta^{\mu \nu, \rho \lambda}(x, y, \eta) \Delta_{\rho \lambda, \alpha^{\prime} \beta^{\prime}}^{-1}\left(z^{\prime}, y, \eta\right) j^{\alpha^{\prime} \beta^{\prime}}\left(z^{\prime}\right) \\
& \quad=\frac{m^{2}}{8} \int d^{4} y d^{4} z d^{4} z^{\prime} j^{\alpha \beta}(z) \delta_{[\alpha}^{\rho} \delta_{\beta]}^{\lambda} \delta^{4}(z-y) \Delta_{\rho \lambda, \alpha^{\prime} \beta^{\prime}}^{-1}\left(z^{\prime}, y, \eta\right) j^{\alpha^{\prime} \beta^{\prime}}\left(z^{\prime}\right) \\
& \quad=\frac{m^{2}}{4} \int d^{4} z d^{4} z^{\prime} j^{\alpha \beta}(z) \Delta_{\alpha \beta, \alpha^{\prime} \beta^{\prime}}^{-1}\left(z, z^{\prime}, \eta\right) j^{\alpha^{\prime} \beta^{\prime}}\left(z^{\prime}\right) \\
& \quad=\frac{m^{2}}{4} \int d^{4} k j^{\alpha \beta}(-k) \Delta_{\alpha \beta, \alpha^{\prime} \beta^{\prime}}^{-1}(k) j^{\alpha^{\prime} \beta^{\prime}}(k)
\end{aligned}
$$

where we have used

$$
\frac{1}{2} \int d^{4} z \Delta_{\rho \lambda, \alpha \beta}^{-1}(x, z, \eta) \Delta^{\alpha \beta, \mu \nu}(z, y, \eta)=\delta_{[\rho}^{\mu} \delta_{\lambda]}^{\nu} \delta^{4}(x-y),
$$

in the second line of Eq. (C5). Using $j^{\alpha \beta}=\frac{1}{2} \varepsilon^{\alpha \beta \rho \lambda} F_{\rho \lambda}=\varepsilon^{\alpha \beta \rho \lambda} \partial_{\rho} A_{\lambda}$, we can reexpress the last term of Eq. (C3). Integrating by parts, we obtain 


$$
\frac{m^{2}}{4} \int d^{4} z d^{4} z^{\prime} j^{\alpha \beta}(z) \Delta_{\alpha \beta, \alpha^{\prime} \beta^{\prime}}^{-1}\left(z, z^{\prime}, \eta\right) j^{\alpha^{\prime} \beta^{\prime}}\left(z^{\prime}\right)=\frac{m^{2}}{4} \int d^{4} z d^{4} z^{\prime} \varepsilon^{\rho \lambda \alpha \beta} A_{\lambda}(z) \partial_{\rho}^{z} \partial_{\rho^{\prime}}^{z^{\prime}} \Delta_{\alpha \beta, \alpha^{\prime} \beta^{\prime}}^{-1}\left(z, z^{\prime}, \eta\right) \varepsilon^{\rho^{\prime} \lambda^{\prime} \alpha^{\prime} \beta^{\prime}} A_{\lambda^{\prime}}\left(z^{\prime}\right)
$$

Then we can find

$$
\begin{aligned}
& \frac{m^{2}}{4} \varepsilon^{\rho \lambda \alpha \beta} \partial_{\rho}^{z} \partial_{\rho^{\prime}}^{z^{\prime}} \Delta_{\alpha \beta, \alpha^{\prime} \beta^{\prime}}^{-1}\left(z, z^{\prime}, \eta\right) \varepsilon^{\rho^{\prime} \lambda^{\prime} \alpha^{\prime} \beta} \\
& \quad=\frac{m^{2}}{4} \varepsilon^{\rho \lambda \alpha \beta} \int d^{4} k \Delta_{\alpha \beta, \alpha^{\prime} \beta^{\prime}}^{-1}(k) k_{\rho} k_{\rho^{\prime}} e^{i k \cdot\left(z-z^{\prime}\right)} \varepsilon^{\rho^{\prime} \lambda^{\prime} \alpha^{\prime} \beta^{\prime}} \\
& \quad=\frac{m^{2}}{4} \varepsilon^{\rho \lambda \alpha \beta} \int d^{4} k \frac{1}{k^{2}}\left(\eta_{\alpha\left[\alpha^{\prime}\right.} \eta_{\left.\beta^{\prime}\right] \beta}-(1-\eta) \frac{k_{[\alpha} k_{\left[\alpha^{\prime}\right.} \eta_{\left.\left.\beta^{\prime}\right] \beta\right]}}{k^{2}}\right) k_{\rho} k_{\rho^{\prime}} e^{i k \cdot\left(z-z^{\prime}\right)} \varepsilon^{\rho^{\prime} \lambda^{\prime} \alpha^{\prime} \beta^{\prime}} \\
& \quad=\frac{m^{2}}{4} \varepsilon^{\rho \lambda \alpha \beta} \int d^{4} k \frac{1}{k^{2}} \eta_{\alpha\left[\alpha^{\prime}\right.} \eta_{\left.\beta^{\prime}\right] \beta} k_{\rho} k_{\rho^{\prime}} e^{i k \cdot\left(z-z^{\prime}\right)} \varepsilon^{\rho^{\prime} \lambda^{\prime} \alpha^{\prime} \beta^{\prime}} \\
& \quad=-m^{2} \int d^{4} k \frac{1}{k^{2}}\left(k^{2} \eta^{\lambda \lambda^{\prime}}-k^{\lambda} k^{\lambda^{\prime}}\right) e^{i k \cdot\left(z-z^{\prime}\right)} .
\end{aligned}
$$

As a consequence, the "effective" action from the quadratic part at the tree level (where the degrees of freedom of $B$ field is integrated out) is given as follows:

$$
\begin{aligned}
\mathcal{S}_{\text {eff }} & =-\frac{1}{4} F^{\mu \nu} F_{\mu \nu}+m^{2} \int d^{4} z d^{4} z^{\prime} A_{\lambda}(z) \int d^{4} k \frac{1}{k^{2}}\left(k^{2} \eta^{\lambda \lambda^{\prime}}-k^{\lambda} k^{\lambda^{\prime}}\right) e^{i k \cdot\left(z-z^{\prime}\right)} A_{\lambda^{\prime}}\left(z^{\prime}\right) \\
& =-\frac{1}{4} F^{\mu \nu} F_{\mu \nu}+m^{2} \int d^{4} k A^{\lambda}(k) A_{\lambda}(-k)-m^{2} \int d^{4} k \frac{(k \cdot A(-k))(k \cdot A(k))}{k^{2}} .
\end{aligned}
$$

[1] S. Weinberg, Phys. Rev. Lett. 19, 1264 (1967).

[2] A. Salam, in Elementary Particle Physics: Relativistic Groups and Analyticity, edited by N. Svartholm (Almqvist and Wiksell, Sweden, 1968).

[3] S. Glashow, Nucl. Phys. 22, 579 (1961).

[4] G. Aad et al. (ATLAS collaboration), Phys. Lett. B 716, 1 (2012).

[5] S. Chatrchyan et al. (CMS collaboration), Phys. Lett. B 716, 30 (2012).

[6] D. J. Gross and F. Wilczek, Phys. Rev. Lett. 30, 1343 (1973).

[7] H. D. Politzer, Phys. Rev. Lett. 30, 1346 (1973).

[8] E. Reya, Phys. Rep. 69, 195 (1981).

[9] S. Coleman and D. J. Gross, Phys. Rev. Lett. 31, 851 (1973).

[10] A. Zee, Phys. Rev. D 7, 3630 (1973).

[11] J. Frankel and J. C. Taylor, Nucl. Phys. B109, 439 (1976).

[12] D. J. Gross, Rev. Mod. Phys. 77, 837 (2005).

[13] G. 't Hooft, Nucl. Phys. B254, 11 (1985).

[14] C. Patrignani et al. (Particle Data Group), Chin. Phys. C 40, 100001 (2016).

[15] J. C. Collins and M. J. Perry, Phys. Rev. Lett. 34, 1353 (1975); G. Baym and S. A. Chin, Phys. Lett. B62, 241 (1976); B. A. Freedman and L. D. McLerran, Phys. Rev. D 16, 1169 (1977); G. Chapline and M. Nauenberg, Phys. Rev.
D 16, 450 (1977); E. V. Surayak, Phys. Lett. 78B, 150 (1978); O. K. Kalashnikov and V. V. Klimpv, Phys. Lett. 88B, 328 (1979); J. I. Kapusta, Nucl. Phys. B148, 461 (1979).

[16] J. Adams et al. (STAR Collaboration), Nucl. Phys. A757, 102 (2005); K. Adcox et al. (PHENIX Collaboration), Nucl. Phys. A757, 184 (2005); I. Arsene et al. (BRAHMS Collaboration), Nucl. Phys. A757, 1 (2005); B. B. Back et al. (PHOBOS Collaboration), Nucl. Phys. A757, 28 (2005).

[17] B. Müller, J. Schukraft, and B. Wyslouch, Annu. Rev. Nucl. Part. Sci. 62, 361 (2012).

[18] D. Kharzeev and A. Zhitnitski, Nucl. Phys. A797, 67 (2007).

[19] D. E. Kharzeev, L. D. McLerran, and H. J. Warringa, Nucl. Phys. A803, 227 (2008).

[20] K. Fukushima, D. Kharzeev, and H. J. Kharzeev, Phys. Rev. D 78, 074033 (2008).

[21] Y. Akamatsu and N. Yamamoto, Phys. Rev. D 90, 125031 (2014).

[22] E. Braaten and R. D. Pisarski, Phys. Rev. Lett. 64, 1338 (1990).

[23] R. Kobes, G. Kunstatter, and A. Rebhan, Phys. Rev. Lett. 64, 2992 (1990).

[24] A. D. Linde, Phys. Lett. 96B, 289 (1980). 
[25] D. Gross, R. Pisarski, and L. Yaffe, Rev. Mod. Phys. 53, 43 (1981).

[26] T. Furusawa and K. Kikkawa, Phys. Lett. 128B, 218 (1983).

[27] A. Czajka and S. Mrówczyński, Phys. Rev. D 91, 025013 (2015).

[28] P. Arnold, Phys. Rev. D 62, 036003 (2000).

[29] D. Bödeker, Phys. Lett. B 426, 351 (1998).

[30] A. Selikov and M. Gyulassy, Phys. Lett. B 316, 373 (1993).

[31] H. Heiselberg, Phys. Rev. Lett. 72, 3013 (1994).

[32] F. Strocci, Phys. Lett. 62B, 60 (1976).

[33] R. Hagg and D. Kastler, J. Math. Phys. (N.Y.) 5, 848 (1964).

[34] T. Kugo and I. Ojima, Prog. Theor. Phys. Suppl. 66, 1 (1979).

[35] C. S. Fischer, J. Phys. G 32, R253 (2006).

[36] M. Chaichian and K. Nishijima, Eur. Phys. J. C 47, 737 (2006).

[37] S. Joglekar, Ann. Phys. (N.Y.) 83, 427 (1974).

[38] C. E. Vayonakis, Lett. Nuovo Cimento 17, 383 (1976).

[39] B. Lee, C. Quigg, and H. B. Thacker, Phys. Rev. D 16, 1519 (1977).

[40] H. Ruegg and M. Ruiz-Altaba, Int. J. Mod. Phys. A 19, 3265 (2004).

[41] M. Veltman, Nucl. Phys. B7, 637 (1968).

[42] M. Veltman, Nucl. Phys. B21, 288 (1970).

[43] K.-I. Shizuya, Nucl. Phys. B121, 125 (1977).

[44] H. Umezawa and S. Kamefuchi, Nucl. Phys. 23, 399 (1961).

[45] G. Curci and R. Ferrari, Nuovo Cimento A 32, 151 (1976).

[46] I. Ojima, Z. Phys. C 13, 173 (1982).

[47] J. M. Cornwall, Phys. Rev. D 26, 1453 (1982).
[48] J. M. Cornwall, J. Papavassiliou, and D. Binsoi, The Pinch Technique and Its Application to Non-Abelian Gauge Theories (Cambridge University Press, Cambridge, England, 2011).

[49] M. Balu and G. Thompson, Ann. Phys. (N.Y.) 205, 130 (1991).

[50] D. Birmingham, M. Balu, M. Rakowski, and G. Thompson, Phys. Rep. 209, 129 (1991).

[51] J. C. Baez, Lect. Notes Phys. 543, 25 (2000).

[52] G. Horowitz and M. Srednicki, Commun. Math. Phys. 130, 83 (1990).

[53] M. Kalb and P. Ramond, Phys. Rev. D 9, 2273 (1974).

[54] D. Mukhopadhyay, J.-e. Alam, and R. Kumar, arXiv:2001.03947.

[55] E. Cremmer and J. Scherk, Nucl. Phys. B72, 117 (1974).

[56] T. J. Allen, M. J. Bowick, and A. Lahiri, Mod. Phys. Lett. A 06, 559 (1991).

[57] A. Lahiri, Phys. Rev. D 63, 105002 (2001).

[58] J. Thierry-Mieg and L. Baulieu, Nucl. Phys. B197, 477 (1982); B228, 259 (1983).

[59] A. Lahiri, Phys. Rev. D 55, 5045 (1997).

[60] R. Kumar and R. P. Malik, Eur. Phys. J. C 71, 1710 (2011).

[61] R. Kumar and R. P. Malik, Euro. Phys. Lett. 94, 11001 (2011).

[62] M. Laine and A. Vuorinen, Basics of Thermal Field Theory (Springer, Switzerland, 2016).

[63] M. Abramowtiz and I. A. Stegun, Handbook of Mathematical Functions with Formulas, Graphs and Mathematical Tables (Dover, New York, 1972). 